\title{
Homogeneous Triebel-Lizorkin Spaces on Stratified Lie Groups
}

\author{
Guorong Hu \\ Graduate School of Mathematical Sciences, The University of Tokyo, 3-8-1 Komaba, Mekuro-ku, Tokyo 153-8914, Japan \\ Correspondence should be addressed to Guorong Hu; hugr@ms.u-tokyo.ac.jp
}

Received 7 January 2013; Accepted 3 March 2013

Academic Editor: Józef Banaś

Copyright (c) 2013 Guorong Hu. This is an open access article distributed under the Creative Commons Attribution License, which permits unrestricted use, distribution, and reproduction in any medium, provided the original work is properly cited.

Homogeneous Triebel-Lizorkin spaces with full range of parameters are introduced on stratified Lie groups in terms of LittlewoodPaley-type decomposition. It is shown that the scale of these spaces is independent of the choice of Littlewood-Paley-type decomposition and the sub-Laplacian used for the construction of the decomposition. Some basic properties of these spaces are given. As the main result of this paper, boundedness of a class of singular integral operators on these function spaces is obtained.

\section{Introduction}

In recent years there were several efforts of extending Besov and Triebel-Lizorkin spaces from Euclidean spaces to other domains and non-isotropic settings. In particular, Han et al. [1] developed a theory of these function spaces on spaces of homogenous type with the additional reverse doubling property. That setting is quite general and includes for example Lie groups of polynomial growth. However, the high level of generality imposes restrictions on the possible values of the parameters of the function spaces.

For the purpose of studying subelliptic regularity, Folland [2] introduced fractional Sobolev spaces and Lipschitz spaces on stratified Lie groups. Later, Folland and Stein [3] established the theory of Hardy spaces on general homogeneous groups. Besov spaces on stratified Lie groups were first introduced by Saka [4], by means of the heat semigroup associated to the sub-Laplacian. Recently, Führ and Mayeli [5] introduced homogeneous Besov spaces on stratified Lie groups in terms of Littlewood-Paley-type decomposition and established wavelet characterization of them. However, the integrability parameter $p$ and the summability parameter $q$ of the function spaces studied in both $[4,5]$ are restricted to be no less than 1. Moreover, systematic treatment of TriebelLizorkin spaces on stratified Lie groups can not be found in the literature, to our best knowledge.

The purpose of this paper is to introduce and study homogeneous Triebel-Lizorkin spaces with full range of parameters on stratified Lie groups. Motivated by [5], we define these function spaces via Littlewood-Paley-type decomposition. We find that a helpful way to treat the case that either the integrability parameter $p$ or the summability parameter $q$ is less than 1 is to take the Peetre type maximal function into consideration. With the help of the almost orthogonality estimate on stratified Lie groups (see Lemma 2), we show that our definition of homogeneous Triebel-Lizorkin spaces is independent of the choice of the Littlewood-Paley-type decomposition and the sub-Laplacian used for the construction of the decomposition. Thus, these function spaces reflect of properties of the group, not of the sub-Laplacian used for the construction of the decomposition.

Singular integral theory is a powerful tool for the study of partial differential equations. The $L^{p}$-boundedness of convolution operators with homogeneous distribution kernels on Lie groups endowed with suitable homogeneous structure was proved by Knapp and Stein [6] (for $p=2$ ) and Korányi and Vági [7] (for $1<p<\infty$ ). In Section 4 of this paper, we prove the boundedness on homogeneous Triebel-Lizorkin spaces of a class of convolution type singular integral operators on stratified Lie groups, which includes convolution operators with homogeneous distribution kernels.

This paper is organized as follows. After reviewing some basic notions concerning stratified Lie groups and their associated sub-Laplaicans in Section 2, in Section 3 we introduce homogeneous Triebel-Lizorkin spaces $\dot{F}_{p, q}^{\alpha}(G)$ on stratified Lie groups, and give some basic properties of them. In Section 4 we show the $\dot{F}_{p, q}^{\alpha}(G)$-boundedness of a class of convolution singular integral operators. Throughout this 
paper the letter $C$ will denote a positive constant which is independent of the main variables involved but whose value may differ from line to line. The notation $a \lesssim b$ or $b \gtrsim a$ for some variable quantities $a$ and $b$ means that $a \leq C b$ for some constant $C>0 ; a \sim b$ stands for $a \lesssim b \lesssim a$. We agree that the set $\mathbb{N}$ of natural numbers contains 0 .

\section{Preliminaries}

In this section we briefly review the basic notions concerning stratified Lie groups and their associated sub-Laplacians. For more details we refer the reader to the monograph by Folland and Stein [3]. A Lie group $G$ is called a stratified Lie group if it is connected and simply connected, and its Lie algebra $\mathfrak{g}$ may be decomposed as a direct sum $\mathfrak{g}=V_{1} \oplus \cdots \oplus V_{m}$, with $\left[V_{1}, V_{k}\right]=V_{k+1}$ for $1 \leq k \leq m-1$ and $\left[V_{1}, V_{m}\right]=0$. Such a group $G$ is clearly nilpotent, and thus it may be identified with $\mathfrak{g}$ (as a manifold) via the exponential map exp $: \mathfrak{g} \rightarrow G$. Examples of stratified Lie groups include Euclidean spaces $\mathbb{R}^{n}$ and the Heisenberg group $\mathbb{H}^{n}$.

The algebra $\mathfrak{g}$ is equipped with a family of dilations $\left\{\delta_{t}\right.$ : $t>0\}$ which are the algebra automorphisms defined by

$$
\delta_{t}\left(\sum_{j=1}^{m} X_{j}\right)=\sum_{j=1}^{m} t^{j} X_{j} \quad\left(X_{j} \in V_{j}\right) .
$$

Under our identification of $G$ with $\mathfrak{g}, \delta_{t}$ may also be viewed as a map $G \rightarrow G$. We generally write $t x$ instead of $\delta_{t}(x)$, for $x \in G$. We shall denote by

$$
\Delta=\sum_{j=1}^{m} j\left[\operatorname{dim}\left(V_{j}\right)\right]
$$

the homogeneous dimension of $G$.

A homogeneous norm on $G$ is a continuous function $x \mapsto|x|$ from $G$ to $[0, \infty)$ smooth away from 0 (the group identity), vanishing only at 0 , and satisfying $\left|x^{-1}\right|=|x|$ and $|t x|=t|x|$ for all $x \in G$ and $t>0$. Homogeneous norms on $G$ always exist and any two of them are equivalent. We assume $G$ is provided with a fixed homogeneous norm. It satisfies a triangle inequality: there exists a constant $\gamma \geq 1$ such that $|x y| \leq \gamma(|x|+|y|)$ for all $x, y \in G$. If $x \in G$ and $r>0$ we define the ball of radius $r$ about $x$ by $B(x, r)=\left\{y \in G:\left|y^{-1} x\right|<\right.$ $r$. The Lebesgue measure on $\mathfrak{g}$ induces a bi-invariant Haar measure on $G$. As done in [3], we fix the normalization of Haar measure by requiring that the measure of $B(0,1)$ be 1 . We shall denote the measure of any measurable $E \subset G$ by $|E|$. Clearly we have $\left|\delta_{t}(E)\right|=t^{\Delta}|E|$. All integrals on $G$ are with respect to (the normalization of) Haar measure. Convolution is defined by

$$
f * g(x)=\int f(y) g\left(y^{-1} x\right) d y=\int f\left(x y^{-1}\right) g(y) d y .
$$

We consider $\mathfrak{g}$ as the Lie algebra of all left-invariant vector fields on $G$, and fix a basis $X_{1}, \ldots, X_{n}$ of $\mathfrak{g}$, obtained as a union of bases of the $V_{j}$. In particular, $X_{1}, \ldots, X_{v}$, with $v=\operatorname{dim}\left(V_{1}\right)$, is a basis of $V_{1}$. We denote by $Y_{1}, \ldots, Y_{n}$ the corresponding basis for right-invariant vector fields, that is,

$$
Y_{j} f(x)=\left.\frac{d}{d t} f\left(\exp \left(t X_{j}\right) x\right)\right|_{t=0} .
$$

If $I=\left(i_{1}, \ldots, i_{n}\right) \in \mathbb{N}^{n}$ is a multi-index we set $X^{I}=X_{1}^{i_{1}} \cdots X_{n}^{i_{n}}$ and $Y^{I}=Y_{1}^{i_{1}} \cdots Y_{n}^{i_{n}}$. Moreover, we set

$$
|I|=\sum_{k=1}^{n} i_{k}, \quad d(I)=\sum_{k=1}^{n} d_{k} i_{k}
$$

where the integers $d_{1} \leq \cdots \leq d_{n}$ are given according to that $X_{k} \in V_{d_{k}}$. Then $X^{I}$ (resp., $Y^{I}$ ) is a left-invariant (resp., rightinvariant) differential operator, homogeneous of degree $d(I)$, with respect to the dilations $\delta_{t}, t>0$.

A complex-valued function $P$ on $G$ is called a polynomial on $G$ if $P \circ \exp$ is a polynomial on $\mathfrak{g}$. Let $\xi_{1}, \ldots, \xi_{n}$ be the basis for the linear forms on $\mathfrak{g}$ dual to the basis $X_{1}, \ldots, X_{n}$ for $\mathfrak{g}$, and set $\eta_{j}=\xi_{j} \circ \exp ^{-1}$. From our definition of polynomials on $G, \eta_{1}, \ldots, \eta_{n}$ are generators of the algebra of polynomials on $G$. Thus, every polynomial on $G$ can be written uniquely as

$$
P=\sum_{I} a_{I} \eta^{I}, \quad a_{I} \in \mathbb{C}
$$

where all but finitely many of the coefficients vanish, and $\eta^{I}=\eta^{i_{1}} \cdots \eta^{i_{n}}$. A polynomial of the type (6) is called of homogeneous degree $L$, where $L \in \mathbb{N}$, if $d(I) \leq L$ holds for all multi-indices $I$ with $a_{I} \neq 0$. We let $\mathscr{P}$ denote the space of all polynomials on $G$, and let $\mathscr{P}_{L}$ denote the space of polynomials on $G$ of homogeneous degree $L$. Note that $\mathscr{P}_{L}$ is invariant under left and right translations (see [3, Proposition 1.25]). A function $f: G \rightarrow \mathbb{C}$ is said to have vanishing moments of order $L$, if

$$
\forall P \in \mathscr{P}_{L}: \quad \int_{G} f(x) P(x) d x=0,
$$

with the absolute convergence of the integral.

The Schwartz class on $G$ is defined by

$$
\begin{gathered}
\mathcal{S}(G)=\left\{f \in C^{\infty}(G): P \frac{\partial^{|I|} f}{\partial \eta^{i_{1}} \cdots \partial \eta^{i_{n}}} \in L^{\infty}(G),\right. \\
\left.\forall I \in \mathbb{N}^{n}, \forall P \in \mathscr{P}\right\} ;
\end{gathered}
$$

that is, $f \in \mathcal{S}(G)$ if and only if $f \circ \exp \in \mathcal{S}(\mathfrak{g})$. As is indicated in [3, p. 35], $\mathcal{S}(G)$ is a Fréchet space and several different choices of families of norms induce the same topology on $\mathcal{S}(G)$. In this paper, for our purpose we use the family $\left\{\|\cdot\|_{(L, N)}: L, N \in \mathbb{N}\right\}$ of norms given by

$$
\|\phi\|_{(L, N)}=\sup _{d(I) \leq L, x \in G}(1+|x|)^{N}\left(\left|X^{I} \phi(x)\right|+\left|X^{I} \widetilde{\phi}(x)\right|\right) .
$$

Here and in what follows, we use the notation convention $\widetilde{\phi}(x)=\phi\left(x^{-1}\right)$ for any function $\phi: G \rightarrow \mathbb{C}$. The dual space 
$\mathcal{S}^{\prime}(G)$ of $\mathcal{S}(G)$ is the space of tempered distributions on $G$. If $f \in \mathcal{S}^{\prime}(G)$ and $\phi \in \mathcal{S}(G)$ we shall denote the evaluation of $f$ on $\phi$ by $\langle f, \phi\rangle$.

Using the above conventions for the choice of the basis $X_{1}, \ldots, X_{n}$, and $v=\operatorname{dim}\left(V_{1}\right)$, the sub-Laplacian is defined by $\mathscr{L}=-\sum_{j=1}^{\nu} X_{j}^{2}$. When restricted to smooth functions with compact support, $\mathscr{L}$ is essentially self-adjoint. Its closure has domain $\mathscr{D}=\left\{u \in L^{2}(G): \mathscr{L} u \in L^{2}(G)\right\}$, where $\mathscr{L} u$ is taken in the sense of distributions. We denote this extension still by the symbol $\mathscr{L}$. By the spectral theorem, $\mathscr{L}$ admits a spectral resolution

$$
\mathscr{L}=\int_{0}^{\infty} \lambda d E(\lambda),
$$

where $d E(\lambda)$ is the projection measure. If $m$ is a bounded Borel measurable function on $[0, \infty)$, the operator

$$
m(\mathscr{L})=\int_{0}^{\infty} m(\lambda) d E(\lambda)
$$

is bounded on $L^{2}(G)$, and commutes with left translations. Thus, by the Schwartz kernel theorem, there exists a tempered distribution $M$ on $G$ such that

$$
m(\mathscr{L}) f=f * M, \quad \forall f \in \mathcal{S}(G) .
$$

Note that the point $\lambda=0$ may be neglected in the spectral resolution, since the projection measure of $\{0\}$ is zero (see $[8$, p. 76]). Consequently we should regard $m$ as functions on $\mathbb{R}^{+} \equiv(0, \infty)$ rather than on $[0, \infty)$.

Let $\mathcal{S}\left(\mathbb{R}^{+}\right)$denote the space of restrictions to $\mathbb{R}^{+}$of functions in $\mathcal{S}(\mathbb{R})$. An important fact proved by Hulanicki [9] is as in the following lemma.

Lemma 1. If $m \in S\left(\mathbb{R}^{+}\right)$then the distribution kernel $M$ of $m(\mathscr{L})$ is in $\mathcal{S}(G)$.

Moreover, from the proof of [10, Corollary 1] we see that if $m$ is a function in $\mathcal{S}\left(\mathbb{R}^{+}\right)$which vanishes identically near the origin, then $M$ is a Schwartz function with all moments vanishing.

In the sequel, if not other specified, we will generally use Greak alphabets with hats to denote functions in $\mathcal{S}\left(\mathbb{R}^{+}\right)$, and use Greek alphabets without hats to denote the associated distribution kernels; for example, for $\widehat{\phi} \in \mathcal{S}\left(\mathbb{R}^{+}\right)$we shall denote by $\phi$ the distribution kernel of the operator $\widehat{\phi}(\mathscr{L})$, where $\mathscr{L}$ is a sub-Laplacian fixed in the context.

\section{Homogeneous Triebel-Lizorkin Spaces on Stratified Lie Groups}

For any function $h$ on $G$ and $t>0$, we define the $L^{1}$ normalized dilation of $h$ by

$$
D_{t} h(x)=t^{\Delta} h(t x) .
$$

Before we introduce the homogeneous Triebel-Lizorkin spaces on stratified Lie groups, we prove the following basic estimate, which is a generalization of [11, Lemma B.1] and will be frequently used throughout this paper.
Lemma 2. Let $L, N \in \mathbb{N}$ with $N \geq L+\Delta+2$. Suppose both $\phi, \psi \in \mathcal{S}(G)$ have vanishing moments of order $L$. Then there exists a constant $C>0$ such that for all $j, \ell \in \mathbb{Z}$ and all $x \in G$,

$$
\begin{aligned}
& \left|\left(D_{2^{j}} \phi\right) *\left(D_{2^{\ell}} \psi\right)(x)\right| \\
& \quad \leq C\|\phi\|_{(L+1, N)}\|\psi\|_{(L+1, N)} 2^{-|j-\ell| L} \frac{2^{(j \wedge \ell) \Delta}}{\left(1+2^{j \wedge \ell}|x|\right)^{N}},
\end{aligned}
$$

where $j \wedge \ell:=\min \{j, \ell\}$.

Proof. Using dilations and the facts $\left(D_{2^{j}} \phi\right) *\left(D_{2^{\ell}} \psi\right)(x)=$ $\left(D_{2^{\ell}} \tilde{\psi}\right) *\left(D_{2^{j}} \widetilde{\phi}\right)\left(x^{-1}\right)$ and $\|\phi\|_{(L, N)}=\|\widetilde{\phi}\|_{(L, N)}$ (see $\left.(9)\right)$, we may assume $\ell \geq j=0$. To proceed we follow the idea in the proof of [11, Lemma B.1]. Let $D_{1}=\{y:|y|<1\}$, $D_{2}=\left\{y:|y| \geq 1\right.$ and $\left.\left|x y^{-1}\right| \leq|x| / 2 \gamma\right\}$ and $D_{3}=\{y:$ $|y| \geq 1$ and $\left.\left|x y^{-1}\right|>|x| / 2 \gamma\right\}$. Let $y \mapsto P_{x, \phi}(y)$ be the left Taylor polynomial of $\phi$ at $x$ of homogeneous degree $L$ (see [3, pp. 26-27]). Then using vanishing moments of $\psi$

$$
\begin{aligned}
\mid \phi * & \left(D_{2^{\ell}} \psi\right)(x) \mid \\
& \leq \int\left|\phi\left(x y^{-1}\right)-P_{x, \phi}\left(y^{-1}\right)\right|\left|\left(D_{2^{\ell}} \psi\right)(y)\right| d y \\
& \equiv \int_{D_{1}}+\int_{D_{2}}+\int_{D_{3}} .
\end{aligned}
$$

For $y \in D_{1}$, the stratified Taylor formula (cf. [3, Corollary 1.44]) yields that, with $b$ a suitable positive constant,

$$
\begin{aligned}
& \left|\phi\left(x y^{-1}\right)-P_{x, \phi}\left(y^{-1}\right)\right| \\
& \quad \leqslant|y|^{L+1} \sup _{\substack{|z| \leq b^{L+1}|y| \\
d(I)=L+1}}\left|\left(X^{I} \phi\right)(x z)\right| \\
& \quad \lesssim\|\phi\|_{(L+1, N)} \sup _{|z| \leq b^{L+1}} \frac{|y|^{L+1}}{(1+|x z|)^{N}} \lesssim\|\phi\|_{(L+1, N)} \frac{|y|^{L+1}}{(1+|x|)^{N}},
\end{aligned}
$$

since $2 b^{L+1}(1+|x z|) \geq 2 b^{L+1}+|x z| \geq 2 b^{L+1}+|x| / \gamma-|z| \geq$ $b^{L+1}+|x| / \gamma \gtrsim(1+|x|) / \gamma$ if $|z| \leq b^{L+1}$. Hence we have

$$
\begin{aligned}
\int_{D_{1}} & \leq\|\phi\|_{(L+1, N)}\|\psi\|_{(0, N)} \frac{2^{\ell \Delta}}{(1+|x|)^{N}} \int_{D_{1}} \frac{|y|^{L+1}}{\left(1+2^{\ell}|y|\right)^{N}} d y \\
& =\|\phi\|_{(L+1, N)}\|\psi\|_{(0, N)} \frac{2^{-\ell(L+1)}}{(1+|x|)^{N}} \int_{G} \frac{\left|2^{\ell} y\right|^{L+1}}{\left(1+2^{\ell}|y|\right)^{N}} d\left(2^{\ell} y\right) \\
& \leqslant\|\phi\|_{(L+1, N)}\|\psi\|_{(0, N)} \frac{2^{-\ell(L+1)}}{(1+|x|)^{N}},
\end{aligned}
$$

where for the last inequality we used [3, Corollary 1.17] and that $N-L-1 \geq \Delta+1$. 
For $y \in D_{2}$, we have $|y| \geq|x| / \gamma-\left|x y^{-1}\right| \geq|x| / \gamma-|x| / 2 \gamma=$ $|x| / 2 \gamma$. On the other hand, $|y| \leq \gamma\left(|x|+\left|x y^{-1}\right|\right) \leq \gamma(|x|+$ $|x| / 2 \gamma)=(\gamma+(1 / 2))|x|$. Thus, we have

$$
\begin{gathered}
1+2^{\ell}|y| \geq 2^{\ell}|y| \geq \frac{2^{\ell}(1+|y|)}{2} \geq \frac{2^{\ell}(1+|x|)}{4 \gamma}, \\
|y|^{L} \leq\left(\gamma+\frac{1}{2}\right)^{L}|x|^{L} .
\end{gathered}
$$

Also, we note that by [12, Proposition 20.3.14] the left Taylor polynomial $P_{x, \phi}$ is of the form

$$
\begin{aligned}
& P_{x, \phi}(y) \\
& =\phi(x)+\sum_{h=1}^{L} \sum_{k=1}^{h} \sum_{\substack{1 \leq i_{1}, \ldots, i_{k} \leq n \\
d_{i_{1}}+\cdots+i_{i_{k}}=h}} \frac{\eta_{i_{1}}(y) \ldots \eta_{i_{k}}(y)}{k !} X_{i_{1}} \cdots X_{i_{k}} \phi(x),
\end{aligned}
$$

where the integers $d_{1} \leq \cdots \leq d_{n}$ are given according to that $X_{j} \in V_{d_{j}}$. From these remarks, it follows that

$$
\begin{aligned}
& \int_{D_{2}} \leqslant\|\phi\|_{(L, N)}\|\psi\|_{(0, N)} \\
& \times \int_{D_{2}}\left[\frac{1}{\left(1+\left|x y^{-1}\right|\right)^{N}}+\sum_{0 \leq h \leq L} \frac{|y|^{h}}{(1+|x|)^{N}}\right] \\
& \times \frac{2^{\ell \Delta}}{\left(1+\left|2^{\ell} y\right|\right)^{N}} d y \\
& \leq\|\phi\|_{(L, N)}\|\psi\|_{(0, N)} \int_{D_{2}}\left[\frac{1}{\left(1+\left|x y^{-1}\right|\right)^{N}}+\frac{|x|^{L}}{(1+|x|)^{N}}\right] \\
& \times \frac{2^{\ell \Delta}}{\left[2^{\ell}(1+|x|)\right]^{N}} d y \\
&=\|\phi\|_{(L, N)}\|\psi\|_{(0, N)} \frac{2^{-\ell(N-\Delta)}}{(1+|x|)^{N}} \\
& \times\left[\int_{G} \frac{1}{\left(1+\left|x y^{-1}\right|\right)^{N}} d y\right. \\
&\left.\quad+\frac{|x|^{L}}{(1+|x|)^{N}} \int_{\left|x y^{-1}\right| \leq|x| / 2 \gamma} d y\right]
\end{aligned}
$$

$$
\begin{aligned}
& \leqslant\|\phi\|_{(L, N)}\|\psi\|_{(0, N)} \frac{2^{-\ell(N-\Delta)}}{(1+|x|)^{N}} \\
& \times\left[\int_{G} \frac{1}{(1+|y|)^{N}} d y\right. \\
& \left.+\frac{|x|^{L}}{(1+|x|)^{N}} \int_{|y| \leq \gamma(|x|+|x| / 2 \gamma)} d y\right] \\
& \leqslant\|\phi\|_{(L, N)}\|\psi\|_{(0, N)} \frac{2^{-\ell(N-\Delta)}}{(1+|x|)^{N}}\left[1+\frac{|x|^{L+\Delta}}{(1+|x|)^{N}}\right] \\
& \lesssim\|\phi\|_{(L, N)}\|\psi\|_{(0, N)} \frac{2^{-\ell L}}{(1+|x|)^{N}},
\end{aligned}
$$

where we used that $|\{y:|y| \leq \gamma(|x|+|x| / 2 \gamma)\}| \sim|x|^{\Delta}$ and $N-\Delta>L$.

For $y \in D_{3}$ we have $\left|x y^{-1}\right|>|x| / 2 \gamma$, and, hence

$$
\begin{aligned}
\int_{D_{3}} \leqslant & \|\phi\|_{(L, N)}\|\psi\|_{(0, N)} \\
& \times \int_{D_{3}}\left[\frac{1}{\left(1+\left|x y^{-1}\right|\right)^{N}}+\sum_{0 \leq h \leq L} \frac{|y|^{h}}{(1+|x|)^{N}}\right] \\
& \times \frac{2^{\ell \Delta}}{\left(1+\left|2^{\ell} y\right|\right)^{N}} d y \\
\leq & \|\phi\|_{(L, N)}\|\psi\|_{(0, N)} \frac{2^{\ell \Delta}}{(1+|x|)^{N}} \int_{|y| \geq 1} \frac{|y|^{L}}{\left(1+2^{\ell}|y|\right)^{N}} d y \\
\leq & \|\phi\|_{(L, N)}\|\psi\|_{(0, N)} \frac{2^{-\ell L}}{(1+|x|)^{N}} \int_{G} \frac{\left|2^{\ell} y\right|^{L}}{\left(1+2^{\ell}|y|\right)^{N}} d\left(2^{\ell} y\right) \\
\leq & \|\phi\|_{(L, N)}\|\psi\|_{(0, N)} \frac{2^{-\ell L}}{(1+|x|)^{N}},
\end{aligned}
$$

where for the last inequality we used [3, Corollary 1.17] and $N-L>\Delta+1$.

Combining the above estimates, we arrive at

$$
\left|\phi *\left(D_{2^{\ell}} \psi\right)(x)\right| \leq\|\phi\|_{(L+1, N)}\|\psi\|_{(L+1, N)} 2^{-\ell L} \frac{1}{(1+|x|)^{N}} .
$$

This is exactly what we need.

Let $\mathscr{Z}(G)$ denote the space of Schwartz functions with all moments vanishing. We then consider $\mathscr{Z}(G)$ as a subspace of $\mathcal{S}(G)$, including the topology. It is shown in [5, Lemma 3.3] that $\mathscr{Z}(G)$ is a closed subspace of $\mathcal{S}(G)$, and the topology dual $\mathscr{Z}^{\prime}(G)$ of $\mathscr{Z}(G)$ can be canonically identified with the factor space $\delta^{\prime}(G) / \mathscr{P}$.

We now have the following Calderón type reproducing formula. 
Lemma 3. Suppose $\mathscr{L}$ is a sub-Laplacian on $G$, and $\widehat{\phi} \epsilon$ $\delta\left(\mathbb{R}^{+}\right)$is a function with compact support, vanishing identically near the origin, and satisfying

$$
\sum_{j \in \mathbb{Z}} \widehat{\phi}\left(2^{-2 j} \lambda\right)=1, \quad \forall \lambda \in \mathbb{R}^{+} .
$$

Then for all $g \in \mathscr{Z}(G)$, it holds that

$$
g=\lim _{k \rightarrow \infty} \sum_{|j| \leq k} g *\left(D_{2^{j}} \phi\right),
$$

with convergence in $\mathscr{Z}(G)$. Duality entails that, for all $u \in$ $\mathcal{S}^{\prime}(G) / \mathscr{P}$,

$$
u=\lim _{k \rightarrow \infty} \sum_{|j| \leq k} u *\left(D_{2^{j}} \phi\right)
$$

and the convergence is in $\mathcal{S}^{\prime}(G) / \mathscr{P}$.

Proof. First note that the 2-homogeneity of $\mathscr{L}$ implies that the distribution kernel of $\widehat{\phi}\left(2^{-2 j} \mathscr{L}\right)$ coincides with $D_{2^{j}} \phi$. Let $I \in \mathbb{N}^{n}$ and $N \in \mathbb{N}$ be arbitrarily chosen. Then take $L \in \mathbb{N}$ such that $L \geq N+d(I)+1$. Since both $g$ and $\phi$ are Schwartz functions with all moments vanishing, it follows by Lemma 2 that

$$
\begin{aligned}
(1 & +|x|)^{N}\left|X^{I}\left[g *\left(D_{2^{j}} \phi\right)\right](x)\right| \\
& =2^{d(I) j}(1+|x|)^{N}\left|g *\left[D_{2^{j}}\left(X^{I} \phi\right)\right](x)\right| \\
& \leq C 2^{d(I) j} 2^{-|j| L} 2^{(j \wedge 0) \Delta}\left(1+2^{(j \wedge 0)}|x|\right)^{-(L+\Delta+2)}(1+|x|)^{N} \\
& \leq C 2^{d(I) j} 2^{-|j| L} 2^{(j \wedge 0) \Delta}\left(1+2^{(j \wedge 0)}|x|\right)^{-N}(1+|x|)^{N} \\
& \leq C 2^{-|j|(L-N-d(I))},
\end{aligned}
$$

where the constant $C$ is a suitable multiple of $\|g\|_{(L+1, L+\Delta+2)}\left\|X^{I} \phi\right\|_{(L+1, L+\Delta+2)}$. This implies that $\sum_{j \in \mathbb{Z}}(1+|x|)^{N}\left|X^{I}\left[g *\left(D_{2^{j}} \phi\right)\right](x)\right|$ converges uniformly in $x$, for every $I \in \mathbb{N}^{n}$ and every $N \in \mathbb{N}$. Consequently there exists $h \in \mathcal{S}(G)$ such that $\sum_{|j| \leq k} g *\left(D_{2^{j}} \phi\right)$ converges in the topology of $\mathcal{S}(G)$ to $h$, as $k \rightarrow \infty$. On the other hand, by (23) and the spectral theorem (cf. [13, Theorem VII.2]),

$$
g=\sum_{j \in \mathbb{Z}} \widehat{\phi}\left(2^{-2 j} \mathscr{L}\right) g=\sum_{j \in \mathbb{Z}} g *\left(D_{2^{j}} \phi\right)
$$

holds in $L^{2}$-norm. Therefore, $h=g$, which completes the proof.

Let $\mathscr{A}$ denote the class of all functions $\hat{\phi}$ in $\mathcal{S}\left(\mathbb{R}^{+}\right)$ satisfying

$$
\begin{gathered}
\operatorname{supp} \hat{\phi} \subset\left[2^{-2}, 2^{2}\right], \\
|\widehat{\phi}(\lambda)| \geq C>0 \quad \text { for } \lambda \in\left[\left(\frac{3}{5}\right)^{2},\left(\frac{5}{3}\right)^{2}\right] .
\end{gathered}
$$

Definition 4. Let $\alpha \in \mathbb{R}, 0<p<\infty$ and $0<q \leq \infty$. Let $\mathscr{L}$ be a sub-Laplacian on $G$ and $\widehat{\phi} \in \mathscr{A}$. We define $\dot{F}_{p, q}^{\alpha}(\mathscr{L}, \widehat{\phi})$ as the space of all $f \in \mathcal{S}^{\prime}(G) / \mathscr{P}$ such that

$$
\|f\|_{\dot{F}_{p, q}^{\alpha}(\mathscr{L}, \widehat{\phi})} \equiv\left\|\left(\sum_{j \in \mathbb{Z}}\left(2^{j \alpha}\left|f *\left(D_{2^{j}} \phi\right)\right|\right)^{q}\right)^{1 / q}\right\|_{L^{p}}<\infty,
$$

with the usual modification for $q=\infty$.

We then introduce the Peetre type maximal functions: Given $f \in \mathcal{S}^{\prime}(G), \hat{\phi} \in \delta\left(\mathbb{R}^{+}\right), \mathscr{L}$ a sub-Laplacian, and $a, t>0$, we define

$$
\begin{gathered}
M_{a, t}^{*}(f, \mathscr{L}, \widehat{\phi})(x)=\sup _{y \in G} \frac{\left|f *\left(D_{t^{-1}} \phi\right)(x)\right|}{\left(1+t^{-1}\left|y^{-1} x\right|\right)^{a}} \\
M_{a, t}^{* *}(f, \mathscr{L}, \widehat{\phi})(x)=\sup _{\substack{y \in G \\
1 \leq k \leq \nu}} \frac{\left|X_{k}\left[f *\left(D_{t^{-1}} \phi\right)\right](x)\right|}{\left(1+t^{-1}\left|y^{-1} x\right|\right)^{a}} .
\end{gathered}
$$

Lemma 5. Suppose $\mathscr{L}$ is a sub-Laplacian and $\widehat{\phi} \in \mathscr{A}$. Then for every $a>0$ there is a constant $C>0$ such that for all $f \in \mathcal{S}^{\prime}(G) / \mathscr{P}$, all $j \in \mathbb{Z}$, and all $x \in G$,

$$
M_{a, 2^{-j}}^{* *}(f, \mathscr{L}, \widehat{\phi})(x) \leq C 2^{j} M_{a, 2^{-j}}^{*}(f, \mathscr{L}, \widehat{\phi})(x) .
$$

Proof. Because of (28) it is possible to find a function $\hat{\psi} \in \mathcal{S}\left(\mathbb{R}^{+}\right)$supported in $\left[2^{-2}, 2^{2}\right]$ such that $\sum_{j \in \mathbb{Z}} \widehat{\phi}\left(2^{-2 j} \lambda\right) \widehat{\psi}\left(2^{-2 j} \lambda\right)=1$ for $\lambda \in \mathbb{R}^{+}$. Set $\widehat{\zeta}(\lambda)=$ $\sum_{j=-1}^{1} \widehat{\phi}\left(2^{-2 j} \lambda\right) \widehat{\psi}\left(2^{-2 j} \lambda\right), \lambda \in \mathbb{R}^{+}$. Then $\widehat{\phi}(\lambda)=\widehat{\phi}(\lambda) \widehat{\zeta}(\lambda)$ for all $\lambda \in \mathbb{R}^{+}$. Consequently, for $j \in \mathbb{Z}$ and $1 \leq k \leq \nu$,

$$
\begin{aligned}
\mid X_{k} & {\left[f *\left(D_{2^{j}} \phi\right)\right](y) \mid } \\
= & \left|X_{k}\left[f *\left(D_{2^{j}} \phi\right) *\left(D_{2^{j}} \zeta\right)\right](y)\right| \\
\leq & \int\left|f *\left(D_{2^{j}} \phi\right)(z)\right|\left|\left[X_{k}\left(D_{2^{j}} \zeta\right)\right]\left(z^{-1} y\right)\right| d z \\
= & 2^{j(\Delta+1)} \int\left|f *\left(D_{2^{j}} \phi\right)(z)\right|\left|\left(X_{k} \zeta\right)\left(2^{j}\left(z^{-1} y\right)\right)\right| d z \\
\leq & 2^{j} M_{a, 2^{-j}}^{*}(f, \mathscr{L}, \widehat{\phi})(x) \\
& \times \int 2^{j \Delta}\left(1+2^{j}\left|z^{-1} x\right|\right)^{a}\left(1+2^{j}\left|z^{-1} y\right|\right)^{-a-(\Delta+1)} d z \\
\leq & 2^{j} M_{a, 2^{-j}}^{*}(f, \mathscr{L}, \widehat{\phi})(x)\left(1+2^{j}\left|y^{-1} x\right|\right)^{a},
\end{aligned}
$$

where for the last inequality we used [3, Corollary 1.17] and that $\left(1+2^{j}\left|z^{-1} x\right|\right)^{a}\left(1+2^{j}\left|z^{-1} y\right|\right)^{-a} \leqslant\left(1+2^{j}\left|y^{-1} x\right|\right)^{a}$. Dividing both sides of the above estimate by $\left(1+2^{j}\left|y^{-1} x\right|\right)^{a}$, and then taking the supremum over $y \in G$ and $1 \leq k \leq v$, we obtain the desired estimate.

Lemma 6. Suppose $\mathscr{L}$ is a sub-Laplacian, $\widehat{\phi} \in \mathscr{A}$, and $r>0$. Then there exists a constant $C$ such that for all $f \in \mathcal{S}^{\prime}(G) / \mathscr{P}$, all $j \in \mathbb{Z}$, and all $x \in G$,

$$
M_{a, 2^{-j}}^{*}(f, \mathscr{L}, \widehat{\phi})(x) \leq C\left[M\left(\left|f *\left(D_{2^{j}} \phi\right)\right|^{r}\right)(x)\right]^{1 / r},
$$


where $a=\Delta / r$, and $M$ is the Hardy-Littlewood maximal operator on $G$.

Proof. Let $g \in C^{\infty}(G)$ and $y_{0} \in G$. The stratified mean value theorem (cf. [3, Theorem 1.41]) gives that for every $\delta>0$ and every $y \in G$ with $\left|y^{-1} y_{0}\right|<\delta$,

$$
\begin{aligned}
& \left|g(y)-g\left(y_{0}\right)\right| \\
& \leq C\left|y_{0}^{-1} y\right| \sup _{\substack{|z| \leq b\left|y_{0}^{-1} y\right| \\
1 \leq k \leq \nu}}\left|\left(X_{k} g\right)\left(y_{0} z\right)\right| \\
& \leq C \delta \sup _{\substack{|z| \leq b \delta \\
1 \leq k \leq \nu}}\left|\left(X_{k} g\right)\left(y_{0} z\right)\right|,
\end{aligned}
$$

where $b$ is a suitable positive constant. Hence we have

$$
\begin{aligned}
\left|g\left(y_{0}\right)\right| \leq & C \delta \sup _{\substack{\left|y^{-1} y_{0}\right| \leq b \delta \\
1 \leq k \leq \nu}}\left|X_{k} g(y)\right| \\
& +\delta^{-a}\left(\int_{\left|y^{-1} y_{0}\right| \leq \delta}|g(y)|^{r} d y\right)^{1 / r} .
\end{aligned}
$$

Putting $g=f *\left(D_{2^{j}} \phi\right)$, dividing both sides by $\left(1+2^{j}\left|y_{0}^{-1} x\right|\right)^{a}$, and using Lemma 5 , we have

$$
\begin{aligned}
& \frac{\left|f *\left(D_{2^{j}} \phi\right)\left(y_{0}\right)\right|}{\left(1+2^{j}\left|y_{0}^{-1} x\right|\right)^{a}} \\
& \leq C \delta \sup _{\left|y^{-1} y_{0}\right| \leq b \delta} \frac{\left|X_{k}\left[f *\left(D_{2^{j}} \phi\right)\right](y)\right|}{\left(1+k \leq 2^{j}\left|y^{-1} x\right|\right)^{a}} \frac{\left(1+2^{j}\left|y^{-1} x\right|\right)^{a}}{\left(1+2^{j}\left|y_{0}^{-1} x\right|\right)^{a}} \\
& \quad+\frac{\delta^{-a}}{\left(1+2^{j}\left|y_{0}^{-1} x\right|\right)^{a}}\left(\int_{\left|y^{-1} y_{0}\right| \leq \delta}\left|f *\left(D_{2^{j}} \phi\right)(y)\right|^{r} d y\right)^{1 / r} \\
& \leq C \delta \sup _{\left|y^{-1} y_{0}\right| \leq b \delta}\left(1+2^{j}\left|y^{-1} y_{0}\right|\right)^{a} M_{a, 2^{-j}}^{* *}(f, \mathscr{L}, \widehat{\phi})(x) \\
& \quad+\frac{\delta^{-a}}{\left(1+2^{j}\left|y_{0}^{-1} x\right|\right)^{a}} \\
& \left.\quad \times\left(\int_{\left|y^{-1} x\right| \leq \gamma^{\prime}\left(\delta+\left|y_{0}^{-1} x\right|\right)}\left|f *\left(D_{2^{j}} \phi\right)(y)\right|^{r} d y\right)\right)^{1 / r} \\
& \leq C \delta\left(1+2^{j} b \delta\right)^{a} 2^{j} M_{a, 2^{-j}}^{*}(f, \mathscr{L}, \widehat{\phi})(x) \\
& \quad+\frac{\delta^{-a} \gamma^{a}\left(\delta+\left|y_{0}^{-1} x\right|\right)^{a}}{\left(1+2^{j}\left|y_{0}^{-1} x\right|\right)^{a}}\left[M\left(\left|f *\left(D_{2^{j}} \phi\right)\right|^{r}\right)(x)\right]^{1 / r} \\
& \leq C \varepsilon(1+b \varepsilon)^{a} M_{a, 2^{-j}}^{*}(f, \mathscr{L}, \widehat{\phi})(x) \\
& +\gamma^{a}\left(1+\varepsilon^{-1}\right)^{a}\left[M\left(\left|f *\left(D_{2^{j}} \phi\right)\right|^{r}\right)(x)\right]^{1 / r},
\end{aligned}
$$

where we have set $\delta=2^{-j} \varepsilon$ and used that $\left(1+2^{j} \mathcal{E}^{-1}\left|y_{0}^{-1} x\right|\right) /(1+$ $\left.2^{j}\left|y_{0}^{-1} x\right|\right) \leqslant 1+\varepsilon^{-1}$. Finally, taking $\varepsilon$ sufficiently small (such that $\left.C \varepsilon(1+b \varepsilon)^{a}<1 / 2\right)$, and taking the supremum over $y_{0} \epsilon$ $G$, we get the desired estimate.

Theorem 7. Suppose $\mathscr{L}^{(1)}, \mathscr{L}^{(2)}$ are any two sub-Laplacians on $G$, and $\widehat{\phi}^{(1)}, \widehat{\phi}^{(2)}$ are any two functions in $\mathscr{A}$. Then, for $\alpha \in$ $\mathbb{R}, 0<p<\infty$ and $0<q \leq \infty$, we have the (quasi-)norm equivalence

$$
\|f\|_{\dot{F}_{p, q}^{\alpha}\left(\mathscr{L}^{(1)}, \hat{\phi}^{(1)}\right)} \sim\|f\|_{\dot{F}_{p, q}^{\alpha}\left(\mathscr{L}^{(2)}, \hat{\phi}^{(2)}\right)}, \quad f \in \mathcal{S}^{\prime}(G) / \mathscr{P} .
$$

Proof. First we note that if $a>\Delta / \min \{p, q\}$ then we have

$$
\begin{gathered}
\left\|\left(\sum_{j \in \mathbb{Z}}\left(2^{j \alpha}\left|M_{a, 2^{-j}}^{*}\left(f, \mathscr{L}^{(i)}, \widehat{\phi}^{(i)}\right)\right|\right)^{q}\right)^{1 / q}\right\|_{L^{p}} \\
\sim\left\|\left(\sum_{j \in \mathbb{Z}}\left(2^{j \alpha}\left|f *\left(D_{2^{j}} \phi^{(i)}\right)\right|\right)^{q}\right)^{1 / q}\right\|_{L^{p}}
\end{gathered}
$$

for $i=1,2$, where $\phi^{(i)}$ denotes the distribution kernel of $\widehat{\phi}^{(i)}\left(\mathscr{L}^{(i)}\right)$. Indeed, the direction " $₹$ " of (38) is obvious, and the other direction follows by Lemma 6 and the Fefferman-Stein vector-valued maximal inequality on spaces of homogeneous type (see, e.g., [14]). Thus, to prove (37), it suffices to show that

$$
\begin{aligned}
& \left\|\left(\sum_{j \in \mathbb{Z}}\left(2^{j \alpha}\left|M_{a, 2^{-j}}^{*}\left(f, \mathscr{L}^{(1)}, \widehat{\phi}^{(1)}\right)\right|\right)^{q}\right)^{1 / q}\right\|_{L^{p}} \\
& \sim\left\|\left(\sum_{j \in \mathbb{Z}}\left(2^{j \alpha}\left|M_{a, 2^{-j}}^{*}\left(f, \mathscr{L}^{(2)}, \widehat{\phi}^{(2)}\right)\right|\right)^{q}\right)^{1 / q}\right\|_{L^{p}} .
\end{aligned}
$$

To this end, let $\widehat{\psi}^{(1)}$ be a function in $\mathcal{S}\left(\mathbb{R}^{+}\right)$with support in $\left[2^{-2}, 2^{2}\right]$ such that $\sum_{j \in \mathbb{Z}} \widehat{\phi}^{(1)}\left(2^{-2 j} \lambda\right) \widehat{\psi}^{(1)}\left(2^{-2 j} \lambda\right)=1$ for $\lambda \epsilon$ $\mathbb{R}^{+}$. For $f \in \mathcal{S}^{\prime}(G) / \mathscr{P}$, by Lemma 3 we have

$$
f=\sum_{j \in \mathbb{Z}} f *\left(D_{2^{j}} \phi^{(1)}\right) *\left(D_{2^{j}} \psi^{(1)}\right)
$$

with convergence in $\mathcal{S}^{\prime}(G) / \mathscr{P}$. Here $\psi^{(1)}$ is the distribution kernel of $\widehat{\psi}^{(1)}\left(\mathscr{L}^{(1)}\right)$. Hence, since $\phi^{(2)} \in \mathscr{Z}(G)$, we have the pointwise representation

$$
\begin{aligned}
f *\left(D_{2^{\ell}} \phi^{(2)}\right)(y)= & \sum_{j \in \mathbb{Z}} f *\left(D_{2^{j}} \phi^{(1)}\right) *\left(D_{2^{j}} \psi^{(1)}\right) \\
& *\left(D_{2^{\ell}} \phi^{(2)}\right)(y), \quad \forall y \in G .
\end{aligned}
$$


It follows that

$$
\begin{aligned}
\mid f * & \left(D_{2^{\ell}} \phi^{(2)}\right)(y) \mid \\
\leq & \sum_{j \in \mathbb{Z}} \int\left|f *\left(D_{2^{j}} \phi^{(1)}\right)(z)\right| \\
& \times\left|\left(D_{2^{j}} \psi^{(1)}\right) *\left(D_{2^{\ell}} \phi^{(2)}\right)\left(z^{-1} y\right)\right| d z \\
\leq & \sum_{j \in \mathbb{Z}} M_{a, 2^{-j}}^{*}\left(f, \mathscr{L}^{(1)}, \widehat{\phi}^{(1)}\right)(y) \\
& \times \int\left(1+2^{j}|z|\right)^{a}\left|\left(D_{2^{j}} \psi^{(1)}\right) *\left(D_{2^{\ell}} \phi^{(2)}\right)(z)\right| d z \\
\equiv & \sum_{j \in \mathbb{Z}} M_{a, 2^{-j}}^{*}\left(f, \mathscr{L}^{(1)}, \widehat{\phi}^{(1)}\right)(y) I_{j, \ell} .
\end{aligned}
$$

Since both $\psi^{(1)}$ and $\phi^{(2)}$ are Schwartz functions with all moments vanishing, we can use Lemma 2 to estimate that, with $L$ sufficiently large,

$$
\begin{aligned}
I_{j, \ell} \leq & C \int\left(1+2^{j}|z|\right)^{a} 2^{-|j-\ell| L} 2^{(j \wedge \ell) \Delta} \\
& \times\left(1+2^{j \wedge \ell}|z|\right)^{-(L+\Delta+2)} d z \\
\leq & C \int\left(1+2^{j}|z|\right)^{a} 2^{-|j-\ell| L} 2^{(j \wedge \ell) \Delta} \\
& \times\left(1+2^{j \wedge \ell}|z|\right)^{-(a+\Delta+1)} d z \\
\leq & C 2^{-|j-\ell|(L-a)} \int 2^{(j \wedge \ell) \Delta}\left(1+2^{j \wedge \ell}|z|\right)^{-(\Delta+1)} d z \\
\leq & C 2^{-|j-\ell|(L-a)} .
\end{aligned}
$$

Here, the constant $C$ is a suitable multiple of $\left\|\psi^{(1)}\right\|_{(L+1, L+\Delta+2)}\left\|\phi^{(2)}\right\|_{(L+1, L+\Delta+2)}$. On the other hand we observe that

$$
\begin{aligned}
M_{a, 2^{-j}}^{*} & \left(f, \mathscr{L}^{(1)}, \widehat{\phi}^{(1)}\right)(y) \\
\leq & M_{a, 2^{-j}}^{*}\left(f, \mathscr{L}^{(1)}, \widehat{\phi}^{(1)}\right)(x)\left(1+2^{j}\left|y^{-1} x\right|\right)^{a} \\
\lesssim & M_{a, 2^{-j}}^{*}\left(f, \mathscr{L}^{(1)}, \widehat{\phi}^{(1)}\right)(x) \\
& \times\left(1+2^{\ell}\left|y^{-1} x\right|\right)^{a} \max \left(1,2^{(j-\ell) a}\right) .
\end{aligned}
$$

Putting these estimates into (42), multiplying both sides by $2^{\ell \alpha}$, dividing both sides by $\left(1+2^{\ell}\left|y^{-1} x\right|\right)^{a}$ and then taking the supremum over $y \in G$, we obtain

$$
\begin{aligned}
& 2^{\ell \alpha} M_{a, 2^{-\ell}}^{*}\left(f, \mathscr{L}^{(2)}, \widehat{\phi}^{(2)}\right)(x) \\
& \quad \leq \sum_{j \in \mathbb{Z}} 2^{-|j-\ell|(L-2 a-|\alpha|)} 2^{j \alpha} M_{a, 2^{-j}}^{*}\left(f, \mathscr{L}^{(1)}, \widehat{\phi}^{(1)}\right)(x) .
\end{aligned}
$$

In view of $[15$, Lemma 2], taking $L>2 a+|\alpha|$ in the above inequality yields the direction " $\lesssim$ ” of (39). By symmetricity, (39) holds, and the proof is complete.
Remark 8. From Theorem 7 we see that the space $\dot{F}_{p, q}^{\alpha}(\mathscr{L}, \widehat{\phi})$ is actually independent of the choice of $\mathscr{L}$ and $\hat{\phi}$. Thus, in what follows we don't specify the choice of $\mathscr{L}$ and $\widehat{\phi}$ and write $\dot{F}_{p, q}^{\alpha}(G)$ instead of $\dot{F}_{p, q}^{\alpha}(\mathscr{L}, \widehat{\phi})$. Henceforth we shall fix any subLaplacian $\mathscr{L}$. Moreover, for the sake of briefness, we will write $M_{a, t}^{*}(f, \widehat{\phi})(x)$ instead of $M_{a, t}^{*}(f, \mathscr{L}, \widehat{\phi})(x)$.

Proposition 9. For $\alpha \in \mathbb{R}, 0<p<\infty$ and $0<q \leq \infty$, one has the continuous inclusion maps $\mathscr{Z}(G) \hookrightarrow \dot{F}_{p, q}^{\alpha}(G) \hookrightarrow$ $\mathcal{S}^{\prime}(G) / \mathscr{P}$.

Proof. Let $g \in \mathscr{Z}(G)$ and $\hat{\phi} \in \mathscr{A}$. Choose $N \geq(\Delta+1) / p$ and $L \geq N+|\alpha|+1$. Since both $g$ and $\phi$ are Schwartz functions with all moments vanishing, it follows by Lemma 2 that

$$
\begin{aligned}
\mid g * & \left(D_{2^{j}} \phi\right)(x) \mid \\
& \leq C\|g\|_{(L+1, L+\Delta+2)} 2^{-|j| L} 2^{(j \wedge 0) \Delta}\left(1+2^{j \wedge 0}|x|\right)^{-(L+\Delta+2)} \\
& \leq C\|g\|_{(L+1, L+\Delta+2)} 2^{-|j| L} 2^{(j \wedge 0) \Delta}\left(1+2^{j \wedge 0}|x|\right)^{-N} \\
& \leq C\|g\|_{(L+1, L+\Delta+2)} 2^{-|j|(L-N)}(1+|x|)^{-N}
\end{aligned}
$$

for all $j \in \mathbb{Z}$ and all $x \in G$, where the constant $C$ is a suitable multiple of $\|\phi\|_{(L+1, L+\Delta+2)}$. This together with [3, Corollary 1.17] give that

$$
\begin{aligned}
\|g\|_{\dot{F}_{p, q}^{\alpha}(G)} & \leqslant\|g\|_{(L+1, L+\Delta+2)}\left(\sum_{j \in \mathbb{Z}} 2^{-|j|(L-N-|\alpha|) q}\right)^{1 / q} \\
& \leqslant\|g\|_{(L+1, L+\Delta+2)},
\end{aligned}
$$

which implies that $\mathscr{Z}(G) \hookrightarrow \dot{F}_{p, q}^{s}(G)$ continuously.

Now we show the other embedding. Let $f \in \dot{F}_{p, q}^{\alpha}(G)$ and take any $\psi \in \mathscr{Z}(G)$. Let $\widehat{\phi} \in \mathscr{A}$, and then let $\widehat{\zeta} \epsilon$ $\mathcal{S}\left(\mathbb{R}^{+}\right)$be a function with support in $\left[2^{-2}, 2^{2}\right]$ such that $\sum_{j \in \mathbb{Z}} \widehat{\phi}\left(2^{-2 j} \lambda\right) \widehat{\zeta}\left(2^{-2 j} \lambda\right)=1$ for $\lambda \in \mathbb{R}^{+}$. Then by Lemma 3 we have

$$
\begin{aligned}
|\langle f, \psi\rangle| & =\left|\left\langle\sum_{j \in \mathbb{Z}} f *\left(D_{2^{j}} \phi\right) *\left(D_{2^{j}} \zeta\right), \psi\right\rangle\right| \\
& \leq \sum_{j \in \mathbb{Z}}\left|\left\langle f *\left(D_{2^{j}} \phi\right), \psi *\left(D_{2^{j}} \widetilde{\zeta}\right)\right\rangle\right| \\
& \leq \sum_{j \in \mathbb{Z}}\left\|f *\left(D_{2^{j}} \phi\right)\right\|_{L^{\infty}}\left\|\psi *\left(D_{2^{j}} \tilde{\zeta}\right)\right\|_{L^{1}}
\end{aligned}
$$

To proceed we claim that

$$
\left\|f *\left(D_{2^{j}} \phi\right)\right\|_{L^{\infty}} \leq C 2^{j \Delta / p}\left\|f *\left(D_{2^{j}} \phi\right)\right\|_{L^{p}}
$$


for all $0<p<\infty$. Assuming the claim for a moment, it follows from (48) that

$$
\begin{aligned}
|\langle f, \psi\rangle| \lesssim & \left(\sup _{j \in \mathbb{Z}} 2^{j \alpha}\left\|f *\left(D_{2^{j}} \phi\right)\right\|_{L^{p}}\right) \\
& \times \sum_{j \in \mathbb{Z}} 2^{j[(\Delta / p)-\alpha]}\left\|\psi *\left(D_{2^{j}} \widetilde{\zeta}\right)\right\|_{L^{1}} .
\end{aligned}
$$

It is easy to see that

$$
\sup _{j \in \mathbb{Z}} 2^{j \alpha}\left\|f *\left(D_{2^{j}} \phi\right)\right\|_{L^{p}} \lesssim\|f\|_{\dot{F}_{p, q}^{\alpha}(G)} .
$$

To estimate the sum in (50), we note that if we choose $N \geq$ $\Delta+1$ and $L \geq N+(\Delta / p)+|\alpha|+1$ then similarly to (46) we have

$$
\left|\psi *\left(D_{2^{j}} \widetilde{\zeta}\right)(x)\right| \leq C\|\psi\|_{(L+1, L+\Delta+2)} 2^{-|j|(L-N)}(1+|x|)^{-N}
$$

where the constant $C$ is a suitable multiple of $\|\widetilde{\zeta}\|_{(L+1, L+\Delta+2)}$. From this it follows that

$$
\begin{aligned}
& \sum_{j \in \mathbb{Z}} 2^{j(\Delta / p-\alpha)}\left\|\psi *\left(D_{2^{j}} \widetilde{\zeta}\right)\right\|_{L^{1}} \\
& \quad \lesssim \sum_{j \in \mathbb{Z}} 2^{-|j|[L-N-(\Delta / p)-|\alpha|]}\|\psi\|_{(L+1, L+\Delta+2)} \\
& \quad \lesssim\|\psi\|_{(L+1, L+\Delta+2)} .
\end{aligned}
$$

Therefore,

$$
|\langle f, \psi\rangle| \lesssim\|f\|_{\dot{F}_{p, q}^{\alpha}(G)}\|\psi\|_{(L+1, L+\Delta+2)} .
$$

This implies that $\dot{F}_{p, q}^{\alpha}(G) \hookrightarrow \mathcal{S}^{\prime}(G) / \mathscr{P}$ continuously.

We are left with showing the claim. Indeed, if $r>0$ is fixed then by Lemma 6 we have, for all $x \in G$,

$$
\begin{aligned}
\left|f *\left(D_{2^{j}} \phi\right)(x)\right| & \sim \inf _{y \in B\left(x, 2^{-j}\right)} \frac{\left|f *\left(D_{2^{j}} \phi\right)(x)\right|}{\left(1+2^{j}\left|x^{-1} y\right|\right)^{\Delta / r}} \\
& \leq \inf _{y \in B\left(x, 2^{-j}\right)} M_{\Delta / r, 2^{-j}}^{*}(f, \widehat{\phi})(y) \\
& \lesssim \inf _{y \in B\left(x, 2^{-j}\right)}\left[M\left(\left|f *\left(D_{2^{j}} \phi\right)\right|^{r}\right)(y)\right]^{1 / r} .
\end{aligned}
$$

Taking $r<p$ in the above estimate and using HardyLittlewood maximal inequality, we have

$$
\begin{aligned}
\mid f * & \left.\left(D_{2^{j}} \phi\right)(x)\right|^{p} \\
& \leq \frac{1}{\left|B\left(x, 2^{-j}\right)\right|} \int_{B\left(x, 2^{-j}\right)}\left[M\left(\left|f *\left(D_{2^{j}} \phi\right)\right|^{r}\right)(y)\right]^{p / r} d y \\
& \leq 2^{j \Delta} \int\left|f *\left(D_{2^{j}} \phi\right)(y)\right|^{p} d y .
\end{aligned}
$$

Since $x$ is arbitrary, the claim follows.
Since all the necessary tools are developed in the above arguments, the following proposition can be proved in the same manner as its Euclidean counterpart; see, for example, the proof of [16, Theorem 2.3.3].

Proposition 10. For $\alpha \in \mathbb{R}, 0<p<\infty$ and $0<q \leq \infty$, $\dot{F}_{p, q}^{\alpha}(G)$ is a quasi-Banach space.

Let us introduce a class of functions. We say that $f \in$ $\mathscr{R}(G)$, if there exists $\widehat{\phi} \in \mathcal{S}\left(\mathbb{R}^{+}\right)$whose support is compact and which vanishes identically near the origin, and $g \in \mathcal{S}(G)$, such that $f=\widehat{\phi}(\mathscr{L}) g$. Clearly $\mathscr{R}(G) \subset \mathscr{Z}(G)$.

Lemma 11. Let $\alpha \in \mathbb{R}$ and $0<p, q<\infty$. Then $\mathscr{R}(G)$ is dense in $\dot{F}_{p, q}^{\alpha}(G)$. In particular, $\mathscr{Z}(G)$ is dense in $\dot{F}_{p, q}^{\alpha}(G)$.

Proof. Take any $u \in \dot{F}_{p, q}^{\alpha}(G)$ and any $\varepsilon>0$. In the appendix we show that $\dot{F}_{p, q}^{\alpha}(G)$ admits smooth atomic decomposition. By the smooth atomic decomposition, we see that $C_{0}^{\infty}(G) \cap$ $\dot{F}_{p, q}^{\alpha}(G)$ is dense in $\dot{F}_{p, q}^{\alpha}(G)$, for $\alpha \in \mathbb{R}$ and $0<p, q<\infty$. Thus, we can find $g \in C_{0}^{\infty}(G) \cap \dot{F}_{p, q}^{\alpha}(G)$ such that $\|g-u\|_{\dot{F}_{p, q}^{\alpha}(G)}<\varepsilon / 2$. On the other hand, the argument in Step 5 of the proof of [16, Theorem 2.3.3] shows that there exists a sufficiently large $N \in \mathbb{N}$ such that $\left\|g-\sum_{j=-N}^{N} g *\left(D_{2^{j}} \phi\right)\right\|_{\dot{F}_{p, q}^{\alpha}(G)}<\varepsilon / 2$. Now we put

$$
h=\sum_{j=-N}^{N} \widehat{\phi}\left(2^{-2 j} \mathscr{L}\right) g=\sum_{j=-N}^{N} g *\left(D_{2^{j}} \phi\right) .
$$

Then $h \in \mathscr{R}(G)$, and we have

$$
\|h-u\|_{\dot{F}_{p, q}^{\alpha}(G)} \lesssim\|h-g\|_{\dot{F}_{p, q}^{\alpha}(G)}+\|g-u\|_{\dot{F}_{p, q}^{\alpha}(G)}<\varepsilon .
$$

This proves the claimed statement.

We next consider lifting property of $\dot{F}_{p, q}^{\alpha}(G)$. For $\sigma \in \mathbb{R}$, the power $\mathscr{L}^{\sigma}$ is naturally given by

$$
\mathscr{L}^{\sigma} f=\int_{0}^{\infty} \lambda^{\sigma} d E(\lambda) f, \quad f \in \operatorname{Dom}\left(\mathscr{L}^{\sigma}\right) \subset L^{2}(G) .
$$

Remark 12. By [17, Theorem 13.24], we have $\mathscr{R}(G) \quad \mathrm{C}$ $\operatorname{Dom}\left(\mathscr{L}^{\sigma}\right)$ for all $\sigma \in \mathbb{R}$. As a consequence, $\operatorname{Dom}\left(\mathscr{L}^{\sigma}\right) \cap$ $\dot{F}_{p, q}^{\alpha}(G)$ is dense in $\dot{F}_{p, q}^{\alpha}(G)$, for all $\alpha, \sigma \in \mathbb{R}$ and $0<p, q<\infty$.

We now have the lifting property of $\dot{F}_{p, q}^{\alpha}(G)$.

Theorem 13. Let $\alpha, \sigma \in \mathbb{R}$ and $0<p, q<\infty$.

(i) The operator $\mathscr{L}^{\sigma}$, initially defined on $\operatorname{Dom}\left(\mathscr{L}^{\sigma}\right) \cap$ $\dot{F}_{p, q}^{\alpha}(G)$, extends to a continuous operator from $\dot{F}_{p, q}^{\alpha}(G)$ to $\dot{F}_{p, q}^{\alpha-2 \sigma}(G)$.

(ii) Let $T_{\sigma}: \dot{F}_{p, q}^{\alpha}(G) \rightarrow \dot{F}_{p, q}^{\alpha-2 \sigma}(G)$ denote the continuous extension of $\mathscr{L}^{\sigma}$. Then $T_{\sigma}$ is an isomorphism, and $\left\|T_{\sigma} f\right\|_{\dot{F}_{p, q}^{\alpha-2 \sigma}(G)}$ is an equivalent quasi-norm of $\dot{F}_{p, q}^{\alpha}(G)$.

Proof. (i) Let $\hat{\phi} \in \mathscr{A}$. Set $\widehat{\zeta}(\lambda)=\lambda^{\sigma}$ and $\widehat{\psi}(\lambda)=$ $\widehat{\phi}(\lambda) \widehat{\zeta}(\lambda)=\widehat{\phi}(\lambda) \lambda^{\sigma}$. Clearly $\widehat{\psi}$ is also in $\mathscr{A}$, and 
$2^{2 j \sigma} \widehat{\psi}\left(2^{-2 j} \lambda\right)=\widehat{\phi}\left(2^{-2 j} \lambda\right) \widehat{\zeta}(\lambda)$. By [17, Theorem 13.24], we have $\widehat{\phi}\left(2^{-2 j} \mathscr{L}\right) \circ \widehat{\zeta}(\mathscr{L}) \subset\left(\widehat{\phi}\left(2^{-2 j} \cdot\right) \hat{\zeta}(\cdot)\right)(\mathscr{L})$, and moreover

$$
\begin{aligned}
\operatorname{Dom} & \left(\widehat{\phi}\left(2^{-2 j} \mathscr{L}\right) \circ \widehat{\zeta}(\mathscr{L})\right) \\
= & \operatorname{Dom}(\widehat{\zeta}(\mathscr{L})) \cap \operatorname{Dom}\left(\left(\widehat{\phi}\left(2^{-2 j}\right) \widehat{\zeta}(\cdot)\right)(\mathscr{L})\right) \\
= & \operatorname{Dom}\left(\mathscr{L}^{\sigma}\right) \cap L^{2}(G)=\operatorname{Dom}\left(\mathscr{L}^{\sigma}\right) .
\end{aligned}
$$

Hence, for every $f \in \operatorname{Dom}\left(\mathscr{L}^{\sigma}\right)$, we have $2^{2 j \sigma} \widehat{\psi}\left(2^{-2 j} \mathscr{L}\right) f=$ $\widehat{\phi}\left(2^{-2 j} \mathscr{L}\right) \mathscr{L}^{\sigma} f$.

Now let $f \in \operatorname{Dom}\left(\mathscr{L}^{\sigma}\right) \cap \dot{F}_{p, q}^{s}(G)$. By the above remarks, we have $2^{2 j \sigma}\left[f *\left(D_{2^{j}} \psi\right)\right]=\left(\mathscr{L}^{\sigma} f\right) *\left(D_{2^{j}} \phi\right)$. It follows that

$$
\begin{aligned}
\left\|\mathscr{L}^{\sigma} f\right\|_{\dot{F}_{p, q}^{\alpha-2 \sigma}(G)} & \\
& \sim\left\|\left(\sum_{j \in \mathbb{Z}}\left(2^{j(\alpha-2 \sigma)}\left|\left(\mathscr{L}^{\sigma} f\right) *\left(D_{2^{j}} \phi\right)\right|\right)^{q}\right)^{1 / q}\right\|_{L^{p}} \\
& =\left\|\left(\sum_{j \in \mathbb{Z}}\left(2^{j \alpha}\left|f *\left(D_{2^{j}} \psi\right)\right|\right)^{q}\right)^{1 / q}\right\|_{L^{p}} \\
& \sim\|f\|_{\dot{F}_{p, q}^{\alpha}(G)} .
\end{aligned}
$$

Since $\operatorname{Dom}\left(\mathscr{L}^{\sigma}\right) \cap \dot{F}_{p, q}^{\alpha}(G)$ is dense in $\dot{F}_{p, q}^{\alpha}(G)$ (see Remark 12), the mapping $\mathscr{L}^{\sigma}: \operatorname{Dom}\left(\mathscr{L}^{\sigma}\right) \cap \dot{F}_{p, q}^{\alpha}(G) \mapsto \dot{F}_{p, q}^{\alpha}(G)$ extends to a continuous operator from $\dot{F}_{p, q}^{\alpha}(G)$ to $\dot{F}_{p, q}^{\alpha-2 \sigma}(G)$. We denote this extension by $T_{\sigma}: \dot{F}_{p, q}^{\alpha}(G) \mapsto \dot{F}_{p, q}^{\alpha-2 \sigma}(G)$.

(ii) Let us first show that the mapping $T_{\sigma}: \dot{F}_{p, q}^{\alpha}(G) \rightarrow$ $\dot{F}_{p, q}^{\alpha-2 \sigma}(G)$ is injective. Indeed, assume $f \in \dot{F}_{p, q}^{\alpha}(G)$ such that $T_{\sigma} f$ is the zero element of $\dot{F}_{p, q}^{\alpha-2 \sigma}(G)$. By Remark 12 we can find a sequence $f_{\ell}$ in $\operatorname{Dom}\left(\mathscr{L}^{\sigma}\right) \cap \dot{F}_{p, q}^{\alpha}(G)$ which converges in $\dot{F}_{p, q}^{\alpha}(G)$ to $f$. Then applying (i) to $\mathscr{L}^{\sigma}$ yields that $\mathscr{L}^{\sigma} f_{\ell}$ converges in $\dot{F}_{p, q}^{s-2 \sigma}(G)$ to the zero element. Since $\mathscr{L}^{\sigma} f_{\ell} \in$ $\operatorname{Dom}\left(\mathscr{L}^{-\sigma}\right) \cap \dot{F}_{p, q}^{\alpha-2 \sigma}(G)$ and $f_{\ell}=\mathscr{L}^{-\sigma}\left(\mathscr{L}^{\sigma} f_{\ell}\right)$, applying (i) to the operator $\mathscr{L}^{-\sigma}$ we see that $f_{\ell}$ converges in $\dot{F}_{p, q}^{\alpha}(G)$ to the zero element. Therefore, $f$ is the zero element in $\dot{F}_{p, q}^{\alpha}(G)$. This proves that $T_{\sigma}$ is injective.

Next we show that $T_{\sigma}: \dot{F}_{p, q}^{\alpha}(G) \rightarrow \dot{F}_{p, q}^{\alpha-2 \sigma}(G)$ is surjective. Indeed, given $f \in \dot{F}_{p, q}^{\alpha-2 \sigma}(G)$, we let $f_{\ell}$ be a sequence in $\operatorname{Dom}\left(\mathscr{L}^{-\sigma}\right) \cap \dot{F}_{p, q}^{\alpha-2 \sigma}(G)$ which converges in $\dot{F}_{p, q}^{\alpha-2 \sigma}(G)$ to $f$. Then from (i) we see that $\mathscr{L}^{-\sigma} f_{\ell}$ converges in $\dot{F}_{p, q}^{\alpha}(G)$. Denote this limit by $g$. We claim that $T_{\sigma} g=f$. Indeed, since $f_{\ell}=$ $\mathscr{L}^{\sigma}\left(\mathscr{L}^{-\sigma} f_{\ell}\right)$, it follows from (i) that $f_{\ell}$ converges to $T_{\sigma} g$ in $\dot{F}_{p, q}^{\alpha}(G)$. Hence $T_{\sigma} g=f$ in $\dot{F}_{p, q}^{\alpha-2 \sigma}(G)$. This proves that $T_{\sigma}$ is surjective.

The above arguments also show that both $T_{-\sigma} \circ T_{\sigma}$ and $T_{\sigma} \circ T_{-\sigma}$ are identity operators on $\dot{F}_{p, q}^{\alpha}(G)$. Furthermore, by an easy density argument we see that (61) holds for all $f \in$ $\dot{F}_{p, q}^{\alpha}(G)$, provided that $\mathscr{L}^{\sigma}$ in (61) is replaced by $T_{\sigma}$. Thus, $T_{\sigma}$ : $\dot{F}_{p, q}^{\alpha}(G) \rightarrow \dot{F}_{p, q}^{\alpha-2 \sigma}(G)$ is an isomorphism, and $\left\|T_{\sigma} f\right\|_{\dot{F}_{p, q}^{\alpha-2 \sigma}(G)}$ is an equivalent quasi-norm of $\dot{F}_{p, q}^{\alpha}(G)$.
Our next goal is to show the Lusin and Littlewood-Paley function characterizations of $\dot{F}_{p, q}^{\alpha}(G)$. If $\alpha \in \mathbb{R}, 0<q<\infty$, $\lambda>0, b \in \mathbb{R}$, and $u(x, k)$ is a function on $G \times \mathbb{Z}$, we define

$$
\begin{gathered}
g_{q}^{\alpha}(u)(x)=\left(\sum_{k=-\infty}^{\infty}\left(2^{k \alpha}|u(x, k)|\right)^{q}\right)^{1 / q}, \\
G_{\lambda, q}^{\alpha}(u)(x)=\left[\sum_{k=-\infty}^{\infty} \int_{G}\left(2^{k \alpha}|u(y, k)|\right)^{q}\right. \\
\left.\times\left(1+2^{k}\left|y^{-1} x\right|\right)^{-\lambda q} 2^{k \Delta} d y\right]^{1 / q}, \\
S_{b, q}^{\alpha}(u)(x) \\
=\left(\sum_{k=-\infty}^{\infty} \int_{\left|y^{-1} x\right| \leq 2^{b-k}}\left(2^{k \alpha}|u(y, k)|\right)^{q} 2^{(k-b) \Delta} d y\right)^{1 / q} .
\end{gathered}
$$

The following proposition shows that the spaces $\dot{F}_{p, q}^{\alpha}(G)$ are characterized by Lusin and Littlewood-Paley functions.

Proposition 14. Let $\alpha \in \mathbb{R}, 0<p, q<\infty, b \in \mathbb{R}$ and $\lambda>$ $\max \{\Delta / p, \Delta / q\}$. Let $f \in \mathcal{S}^{\prime}(G) / \mathscr{P}$ and $\hat{\phi} \in \mathscr{A}$. Put $u(x, k)=$ $f *\left(D_{2^{k}} \phi\right)(x)$, for $x \in G$ and $k \in \mathbb{Z}$. Then one has

$$
\left\|g_{q}^{\alpha}(u)\right\|_{L^{p}} \sim\left\|S_{b, q}^{\alpha}(u)\right\|_{L^{p}} \sim\left\|G_{\lambda, q}^{\alpha}(u)\right\|_{L^{p}} .
$$

Proof. Step 1 . Show that if $\lambda>\max \{\Delta / p, \Delta / q\}$ then

$$
\left\|g_{q}^{\alpha}(u)\right\|_{L^{p}} \leqslant\left\|G_{\lambda, q}^{\alpha}(u)\right\|_{L^{p}} \leqslant\left\|S_{b, q}^{\alpha}(u)\right\|_{L^{p}} .
$$

Indeed, the proof of the first inequality in (64) is essentially the same as that of [18, Theorem 2.3]. To see the second inequality in (64), one only needs to examine the proofs of Theorems 1, 2 and 4 in [19, Chapter 4] and observe that, although the function $u$ considered in [19] is defined on the half space $\mathbb{R}_{+}^{n+1}=\mathbb{R}^{n} \times(0, \infty)$, the arguments there can also be adapted to functions $u$ which are defined on $G \times \mathbb{Z}$.

Step 2. We prove that $\left\|S_{b, q}^{\alpha}(u)\right\|_{L^{p}} \lesssim\left\|g_{q}^{\alpha}(u)\right\|_{L^{p}}$. First note that, by an argument similar to the proofs of [19, Theorems 1 and 2], it follows that $\left\|S_{b, q}^{\alpha}(u)\right\|_{L^{p}} \sim\left\|S_{0, q}^{\alpha}(u)\right\|$ for every fixed $b \in \mathbb{R}$. Hence, in view of (38), it is enough to show that

$$
\left\|S_{0, q}^{\alpha}(u)\right\|_{L^{p}} \lesssim\left\|\left(\sum_{j \in \mathbb{Z}}\left(2^{j \alpha}\left|M_{\lambda, 2^{-j}}^{*}(f, \widehat{\phi})\right|\right)^{q}\right)^{1 / q}\right\|_{L^{p}} .
$$


But this is a consequence of the following elementary estimate:

$$
\begin{aligned}
& \int_{\left|y^{-1} x\right|<2^{-j}}\left(2^{j \alpha}\left|f *\left(D_{2^{j}} \phi\right)(y)\right|\right)^{q} 2^{j \Delta} d y \\
& \quad \sup _{y \in B\left(x, 2^{-j}\right)} \frac{\left(2^{j \alpha}\left|f *\left(D_{2^{j}} \phi\right)(y)\right|\right)^{q}}{\left(1+2^{j}\left|y^{-1} x\right|\right)^{\lambda q}} \\
& \leq \sup _{y \in G} \frac{\left(2^{j \alpha}\left|f *\left(D_{2^{j}} \phi\right)(y)\right|\right)^{q}}{\left(1+2^{j}\left|y^{-1} x\right|\right)^{\lambda q}} \\
& =\left[2^{j \alpha} M_{\lambda, 2^{-j}}^{*}(f, \widehat{\phi})(x)\right]^{q} .
\end{aligned}
$$

The proof of Proposition 14 is thus complete.

Corollary 15. Let $G$ be a stratified Lie group. Then $\dot{F}_{p, 2}^{0}(G)=$ $H^{p}(G)$ with equivalent (quasi-)norms, for $0<p<\infty$. Here $H^{p}(G)$ are Hardy spaces on $G$.

Proof. In [3, Chapter 7], Folland and Stein proved the characterization of Hardy spaces $H^{p}(G)$ by continuous version Lusin function, for $0<p<\infty$. Note that the arguments in [3, Chapter 7] are still valid if we replace the continuous version Lusin function by discrete version one defined above; see also [20] for a treatment of discrete version Lusin funcion. This fact together with Proposition 14 yield the identification of $\dot{F}_{p, 2}^{0}(G)$ with $H^{p}(G)$ for $0<p<\infty$.

\section{Convolution Singular Integral Operators on $\dot{F}_{p, q}^{\alpha}(G)$}

In this section we study boundedness of convolution singular integral operators on homogeneous Triebel-Lizorkin spaces on stratified Lie groups. Motivated by [21, Section 5.3 in Chapter XIII], we introduce a class of singular convolution kernels as follows.

Definition 16. Let $r$ be a positive integer. A kernel of order $r$ is a distribution $K \in \mathcal{S}^{\prime}(G)$ with the following properties:

(i) $K$ coincides with a $C^{r}$ function $K(x)$ away from the group identity 0 and enjoys the regularity condition:

$$
\left|X^{I} K(x)\right| \leq C_{I}|x|^{-Q-d(I)}, \quad \text { for }|I| \leq r, x \neq 0 .
$$

(ii) $K$ satisfies the cancellation condition: For all normalized bump function $\phi$ and all $R>0$, we have

$$
\left|\left\langle K, \phi^{R}\right\rangle\right| \leq C
$$

where $\phi^{R}(x)=\phi(R x)$, and $C$ is a constant independent of $\phi$ and $R$. Here, by a normalized bump function we mean a function $\phi$ supported in $B(0,1)$ and satisfying

$$
\left|X^{I} \phi(x)\right| \leq 1, \quad \forall|I| \leq N, \forall x \in G,
$$

for some fixed positive integer $N$.

The convolution operator $T$ with kernel of order $r$ is called a singular integral operator of order $r$.
Remark 17. Using [3, Proposition 1.29], it is easy to verify that (67) is equivalent to the following condition:

$$
\left|Y^{I} K(x)\right| \leq C_{I}|x|^{-Q-d(I)}, \quad \text { for }|I| \leq r, x \neq 0 .
$$

Examples of such kernels include the class of distributions which are homogeneous of degree $-\Delta$ (see Folland and Stein [3, p. 11] for definition) and agree with $C^{\infty}$ functions away from 0 . Indeed, assume $K \in \mathcal{S}^{\prime}(G)$ is such a distribution, then it is easy to verify that $K$ satisfies the regularity condition (i) in Definition 16; moreover, from [3, Proposition 6.13] we see that $K$ is a principle value distribution such that $\int_{\varepsilon<|x|<L} K(x) d x=$ 0 for all $0<\varepsilon<L<\infty$. Hence, for every normalized bump function $\phi$, by the homogeneity of $K$ we have

$$
\begin{aligned}
\left|\left\langle K, \phi^{R}\right\rangle\right| & =|\langle K, \phi\rangle| \\
& =\left|\lim _{\varepsilon \rightarrow 0} \int_{\varepsilon<|x|<2} K(x)[\phi(x)-\phi(0)] d x\right| \\
& \leq \int_{|x|<2}|K(x)||\phi(x)-\phi(0)| d x .
\end{aligned}
$$

Using stratified mean value theorem (cf. [3, Theorem 1.41]) and (67)-(69), it is easy to verify that the last integral converges absolutely and is bounded by a constant independent of $\phi$ and $R$. Hence $K$ satisfies the condition (ii) in Definition 16.

Now we state the main result of this section.

Theorem 18. Let $\alpha \in \mathbb{R}, 0<p, q<\infty$, and let $r$ be a positive integer such that $r>\Delta / \min \{p, q\}+|\alpha|+2$. Suppose $T$ is a singular integral operator of order $r$. Then $T$ extends to a bounded operator on $\dot{F}_{p, q}^{\alpha}(G)$.

If $K \in \mathcal{S}^{\prime}(G)$ and $t>0$, we define $D_{t} K$ as the tempered distribution given by $\left\langle D_{t} K, \phi\right\rangle=\left\langle K, \phi\left(t^{-1} \cdot\right)\right\rangle(\forall \phi \in \mathcal{S}(G))$. For the proof of Theorem 18, we will need the following lemma, in which $b$ is the positive constant as in [3, Corollary $1.44]$.

Lemma 19. Let $r$ be a positive integer. Suppose $K$ is a kernel of order $r$, and $\phi$ is a smooth function supported in $B\left(0,1 / 4 \gamma^{2} b^{r}\right)$ and having vanishing moments of order $r-1$. Then, there exists a constant $C>0$ such that for all $j \in \mathbb{Z}$, all $I \in \mathbb{N}^{n}$ with $|I| \leq r$, and all $x \in G$

$$
\begin{aligned}
\max & \left\{\left|X^{I}\left[\phi *\left(D_{2^{j}} K\right)\right](x)\right|,\left|Y^{I}\left[\phi *\left(D_{2^{j}} K\right)\right](x)\right|\right\} \\
& \leq C(1+|x|)^{-\Delta-r} .
\end{aligned}
$$

Moreover, $\phi *\left(D_{2^{j}} K\right)$ have vanishing moments of the same order as $\phi$.

Proof. Recall that the convolution of $\phi \in \mathcal{S}(G)$ with $K \in$ $\mathcal{S}^{\prime}(G)$ is defined by $\phi * K(x):=\left\langle K,\left({ }^{x} \phi\right)^{\sim}\right\rangle$, where ${ }^{x} \phi$ is the function given by ${ }^{x} \phi(z)=\phi(x z)$, and as before $\tilde{f}(x):=$ $f\left(x^{-1}\right)$ for any function $f: G \rightarrow \mathbb{C}$. From $[3$, p. 38] we see that $\phi *\left(D_{2^{j}} K\right)$ are $C^{\infty}$ functions, $j \in \mathbb{Z}$. We claim that for every $x$ with $|x| \leq 1 / 2 \gamma$, the function $z \mapsto\left({ }^{x} \phi\right)^{\sim}(z)$ 
is a normalized bump function multiplied with a constant independent of $x$. Indeed, using the quasi-triangle inequality satisfied by the homogeneous norm it is easy to verify that the function $z \mapsto\left({ }^{x} \phi\right)^{\sim}(z)$ is supported in $B(0,1)$; moreover, since $|x| \leq 1 / 2 \gamma$ and since

$$
Y^{I}=\sum_{\substack{|J| \leq|I| \\ d(J) \geq d(I)}} P_{I, J} X^{J}
$$

where $P_{I J,}$ are polynomials of homogeneous degree $d(J)-d(I)$ (see [3, Proposition 1.29]), we have

$$
\begin{aligned}
\left|X^{I}\left[\left({ }^{x} \phi\right)^{\sim}\right](z)\right| \\
\quad=\left|Y^{I}\left({ }^{x} \phi\right)\left(z^{-1}\right)\right| \\
\quad \lesssim \sum_{\substack{|J| \leq|I| \\
d(J) \geq d(I)}}\left|P_{I, J}\left(z^{-1}\right)\right|\left|X^{J}\left({ }^{x} \phi\right)\left(z^{-1}\right)\right| \\
=\sum_{\substack{|J| \leq|I| \\
d(J) \geq d(I)}}\left|P_{I, J}\left(z^{-1}\right)\right|\left|\left(X^{J} \phi\right)\left(x z^{-1}\right)\right| \leq C_{I} .
\end{aligned}
$$

Here $C_{I}$ is a constant depending on $I$ but not on $x$. Hence the claim is true. The above argument also shows that, for every $x$ with $|x| \leq 1 / 2 \gamma$ and for every $I \in \mathbb{N}^{n},\left[{ }^{x}\left(Y^{I} \phi\right)\right]^{\sim}$ is a normalized bump function multiplied with a constant $C_{I}^{\prime}$ independent of $x$. Thus, by the condition (ii) in Definition 16, there exits a constant $C>0$ such that for all $j \in \mathbb{Z}$, all $I \in \mathbb{N}^{n}$ with $|I| \leq r$, and all $x$ with $|x| \leq 1 / 2 \gamma$

$$
\begin{aligned}
\mid Y^{I}[\phi & \left.*\left(D_{2^{j}} K\right)\right](x) \mid \\
& \left.=\mid\left(Y^{I} \phi\right) *\left(D_{2^{j}} K\right)\right](x) \mid \\
& =\left|\left\langle D_{2^{j}} K,\left[^{x}\left(Y^{I} \phi\right)\right]^{\sim}\right\rangle\right| \\
& =\left|\left\langle K,\left[x\left(Y^{I} \phi\right)\right]^{\sim}\left(2^{-j} \cdot\right)\right\rangle\right| \leq C .
\end{aligned}
$$

From this and [3, Proposition 1.29], we also get that, for all $j \in \mathbb{Z}$, all $I \in \mathbb{N}^{n}$ with $|I| \leq r$, and all $x$ with $|x| \leq 1 / 2 \gamma$

$$
\begin{aligned}
& \left|X^{I}\left[\phi *\left(D_{2^{j}} K\right)\right](x)\right| \\
& \quad \leq C \sum_{\substack{\left|I^{\prime}\right| \leq|I| \\
d\left(I^{\prime}\right) \geq d(I)}}|x|^{d\left(I^{\prime}\right)-d(I)}\left|Y^{I^{\prime}}\left[\phi *\left(D_{2^{j}} K\right)\right](x)\right| \leq C .
\end{aligned}
$$

Let now $|x|>1 / 2 \gamma$. Let $y \in \operatorname{supp} \phi$. Let $I \in \mathbb{N}^{n}$ with $|I| \leq r$, and denote by $\left.\widetilde{P}_{x, Y^{I}\left(D_{2} j\right.} K\right)$ the right Taylor polynomial of $Y^{I}\left(D_{2^{j}} K\right)$ at $x$ of homogeneous degree $r-|I|-1$ (see [3, pp. 26-27]). Then by the right-invariant version of [3, Corollary 1.44], we have

$$
\begin{aligned}
& \left|X^{I}\left(D_{2^{j}} K\right)\left(y^{-1} x\right)-\widetilde{P}_{x, X^{I}\left(D_{2^{j}} K\right)}\left(y^{-1}\right)\right| \\
& \leq C|y|^{r-|I|} \sup _{\substack{|z| \leq b^{r-I I \mid}|y| \\
d(J)=r-|I|}}\left|Y^{J} X^{I}\left(D_{2^{J}} K\right)(z x)\right| .
\end{aligned}
$$

Observe that $D_{2^{j}} K$ satisfies (67) with the bound $C$ independent of $j \in \mathbb{Z}$. Also note that for $y \in \operatorname{supp} \phi$ and $|z| \leq b^{r-|I|}|y|$ we have $z x \in G \backslash\{0\}$. Thus, for all $J$ with $d(J)=r-|I|$ and all $z$ with $|z| \leq b^{r-|I|}|y|$, by using (73) and (67) (with $K$ replaced by $D_{2^{j}} K$ ) we have

$$
\begin{aligned}
& \left|Y^{J} X^{I}\left(D_{2^{j}} K\right)(z x)\right| \\
& \quad \leq \sum_{\substack{\left|J^{\prime}\right| \leq|J| \\
d\left(J^{\prime}\right) \geq d(J)}}\left|P_{J, J^{\prime}}(z x)\right|\left|X^{J^{\prime}+I}\left(D_{2^{j}} K\right)(z x)\right| \\
& \quad \lesssim \sum_{\substack{\left|J^{\prime}\right| \leq|J| \\
d\left(J^{\prime}\right) \geq d(J)}}|z x|^{d\left(J^{\prime}\right)-d(J)}|z x|^{-\Delta-d\left(J^{\prime}+I\right)} \\
& \quad \leq|z x|^{-\Delta-r+|I|-d(I)} .
\end{aligned}
$$

Here, for the second inequality we also used the observation that when $d(J)=r-|I|$ and $\left|J^{\prime}\right| \leq|J|$, we have $\left|J^{\prime}+I\right| \leq$ $|J+I| \leq d(J)+|I|=r-|I|+|I|=r$. Inserting (78) into (77) we obtain

$$
\begin{aligned}
& \left|X^{I}\left(D_{2^{j}} K\right)\left(y^{-1} x\right)-\widetilde{P}_{x, X^{I}\left(D_{2^{j}} K\right)}\left(y^{-1}\right)\right| \\
& \leq C|y|^{r-|I|} \sup _{|z| \leq b^{r-|I|}|y|}|z x|^{-\Delta-r+|I|-d(I)} .
\end{aligned}
$$

Notice that for $|x| \geq 1 / 2 \gamma, y \in \operatorname{supp} \phi$ and $|z| \leq b^{r-|I|}|y|$, we have $|z x| \sim|x|$. Thus, by using the vanishing moments of $\phi$ and (79), we have

$$
\begin{aligned}
& \left|X^{I}\left[\phi *\left(D_{2^{j}} K\right)\right](x)\right| \\
& \quad=\left|\int \phi(y) X^{I}\left(D_{2^{j}} K\right)\left(y^{-1} x\right) d y\right| \\
& \quad \leq \int|\phi(y)|\left|X^{I}\left(D_{2^{j}} K\right)\left(y^{-1} x\right)-\widetilde{P}_{x, X^{I}\left(D_{2^{j}} K\right)}\left(y^{-1}\right)\right| d y \\
& \quad \leq C \sup _{|z| \leq b^{r-|I|}|y|} \int|z x|^{-\Delta-r+|I|-d(I)}|y|^{r-|I|}|\phi(y)| d y \\
& \quad \leq C|x|^{-\Delta-r+|I|-d(I)} \int|y|^{r-|I|}|\phi(y)| d y \\
& \leq C|x|^{-\Delta-r+|I|-d(I)} .
\end{aligned}
$$

Combining (76) and (80), we see that, for all $j \in \mathbb{Z}$, all $I \in \mathbb{N}^{n}$ with $|I|<r$, and all $x \in G$

$$
\left|X^{I}\left[\phi *\left(D_{2^{j}} K\right)\right](x)\right| \leq C(1+|x|)^{-\Delta-r+|I|-d(I)} .
$$


From this and (73), we also get that, for all $j \in \mathbb{Z}$, all $I \in \mathbb{N}^{n}$ with $|I|<r$, and all $x \in G$

$$
\begin{aligned}
& \left|Y^{I}\left[\phi *\left(D_{2^{j}} K\right)\right](x)\right| \\
& \leq \sum_{\substack{\left|I^{\prime}\right| \leq|I| \\
d\left(I^{\prime}\right) \geq d(I)}}\left|P_{I, I^{\prime}}(x)\right| X^{I^{\prime}}\left[\phi *\left(D_{2^{j}} K\right)\right](x) \mid \\
& \quad \leq C \sum_{\substack{\left|I^{\prime}\right| \leq|I| \\
d\left(I^{\prime}\right) \geq d(I)}}|x|^{d\left(I^{\prime}\right)-d(I)}(1+|x|)^{-\Delta-r+\left|I^{\prime}\right|-d\left(I^{\prime}\right)} \\
& \leq C(1+|x|)^{-\Delta-r+|I|-d(I)} .
\end{aligned}
$$

Since $|I| \leq d(I)$, (81) along with (82) yield (72).

It is straightforward to verify that $\phi *\left(D_{2^{j}} K\right)$ have vanishing moments of the same order as $\phi$. The proof of Lemma 19 is therefore complete.

The proof of Theorem 18 also relies on the existence of smooth functions with compact support and having arbitrarily high order vanishing moments.

Lemma 20. Given any nonnegative integer $L$ and any positive number $\delta$, there exists a function $\widehat{\zeta} \in \mathcal{S}\left(\mathbb{R}^{+}\right)$with the following properties:

(i) $|\widehat{\zeta}(\lambda)| \geq C>0$ for $\lambda \in\left[2^{-2\left(k_{0}+1\right)}, 2^{-2\left(k_{0}-1\right)}\right]$, with $k_{0}$ some (large) positive integer;

(ii) $\zeta$ is a Schwartz function on $G$ having vanishing moments of order L;

(iii) $\operatorname{supp} \zeta \subset B(0, \delta)$.

Proof. From the appendix of [22] we see that there exists $\widehat{\theta} \in \mathcal{S}\left(\mathbb{R}^{+}\right)$such that $\hat{\theta}(0)=1$ and $\theta$ has compact support. Now let us define $\widehat{\zeta}(\lambda)=\left(t^{-2} \lambda\right)^{k} \widehat{\theta}\left(t^{-2} \lambda\right), \lambda \in \mathbb{R}^{+}$. Here $t>0$ and $k$ is a nonnegative integer. Then $\zeta(x)=D_{t}\left(\mathscr{L}^{k} \theta\right)(x)=$ $t^{\Delta}\left(\mathscr{L}^{k} \theta\right)(t x)$. Hence, if we take $t, k$ sufficiently large, then (ii) and (iii) follow immediately. Moreover, since $\hat{\theta}(0)=1$, it is easy to see that (i) is also satisfied, provided that $k_{0}$ is sufficiently large.

We are now ready to prove Theorem 18.

Proof of Theorem 1. Choose a function $\widehat{\zeta} \in \mathcal{S}\left(\mathbb{R}^{+}\right)$which satisfies conditions (i)-(iii) in Lemma 20 with $L=r-1$ and $\delta=1 / 4 \gamma^{2} b^{r}$. The condition (i) guarantees the existence of a function $\widehat{\psi} \in \mathcal{S}\left(\mathbb{R}^{+}\right)$with the following properties:

$$
\begin{aligned}
& \operatorname{supp} \widehat{\psi} \subset\left[2^{-2\left(k_{0}+1\right)}, 2^{-2\left(k_{0}-1\right)}\right], \\
& |\widehat{\psi}(\lambda)|>0 \quad \text { for } \lambda \in\left(2^{-2\left(k_{0}+1\right)}, 2^{-2\left(k_{0}-1\right)}\right), \\
& \sum_{j \in \mathbb{Z}} \widehat{\psi}\left(2^{-2 j} \lambda\right) \widehat{\zeta}\left(2^{-2 j} \lambda\right)=1, \quad \forall \lambda \in \mathbb{R}^{+} .
\end{aligned}
$$

Note that $\widehat{\psi}\left(2^{-2 k_{0}} \cdot\right) \in \mathscr{A}$. For $f \in \mathscr{Z}(G)$, by Lemma 3 we have

$$
f=\sum_{j \in \mathbb{Z}} f *\left(D_{2^{j}} \psi\right) *\left(D_{2^{j}} \zeta\right)
$$

with convergence in $\mathscr{Z}(G)$. Let $\widehat{\phi} \in \mathscr{A}$, and let $K$ be the convolution kernel of the operaotor $T$. Then we have the representation

$$
\begin{array}{rl}
f & * K *\left(D_{2^{\ell}} \phi\right) \\
& =\sum_{j \in \mathbb{Z}} f *\left(D_{2^{j}} \psi\right) *\left(D_{2^{j}} \zeta\right) * K *\left(D_{2^{\ell}} \phi\right) \\
& =\sum_{j \in \mathbb{Z}} f *\left(D_{2^{j}} \psi\right) * D_{2^{j}}\left[\zeta *\left(D_{2^{-j}} K\right)\right] *\left(D_{2^{\ell}} \phi\right),
\end{array}
$$

which holds pointwise and also in the sense of $\mathcal{S}^{\prime}(G)$. Since (by Lemma 19$) \zeta *\left(D_{2^{-j}} K\right)$ satisfies the decay condition (72) (with the bound $C$ independent of $j \in \mathbb{Z}$ ) and has vanishing moments of the same order as $\zeta$, from the proof of Lemma 2 we see that

$$
\begin{aligned}
& \left|D_{2^{j}}\left[\zeta *\left(D_{2^{-j}} K\right)\right] *\left(D_{2^{\ell}} \phi\right)(y)\right| \\
& \leq C 2^{-|j-\ell|(r-2)} \frac{2^{(j \wedge \ell) \Delta}}{\left(1+2^{j \wedge \ell}|y|\right)^{r+\Delta}} .
\end{aligned}
$$

This together with (85) gives that

$$
\begin{array}{rl}
\mid f * & K *\left(D_{2^{\ell}} \phi\right)(x) \mid \\
\leq & \sum_{j \in \mathbb{Z}} \int\left|f *\left(D_{2^{j}} \psi\right)(z)\right| \\
& \times\left|D_{2^{j}}\left[\zeta *\left(D_{2^{-j}} K\right)\right] *\left(D_{2^{\ell}} \phi\right)\left(z^{-1} x\right)\right| d z \\
\leq & \sum_{j \in \mathbb{Z}} 2^{-|j-\ell|(r-2)} \int \frac{2^{(j \wedge \ell) \Delta}\left|f *\left(D_{2^{j}} \psi\right)(z)\right|}{\left(1+2^{j \wedge \ell}\left|z^{-1} x\right|\right)^{r+\Delta}} d z \\
\lesssim & \sum_{j \in \mathbb{Z}} 2^{-|j-\ell|(r-2-a)}\left[\sup _{z \in G} \frac{\left|f *\left(D_{2^{j}} \psi\right)(z)\right|}{\left(1+2^{j}\left|z^{-1} x\right|\right)^{a}}\right] \\
& \times \int \frac{2^{(j \wedge \ell) \Delta}}{\left(1+2^{j \wedge \ell}\left|z^{-1} x\right|\right)^{r+\Delta-a}} d z,
\end{array}
$$

where for the last inequality we used that $\left(1+2^{j \wedge \ell}\left|z^{-1} x\right|\right)^{-a} \leq$ $2^{|j-\ell| a}\left(1+2^{j}\left|z^{-1} x\right|\right)^{-a}$. By the hypothesis we can choose $a$ such that $a>\Delta / \min \{p, q\}$ and $r-2-a-|\alpha|>0$. From [3, Corollary 1.17] we see that the last integral converges absolutely. Consequently, we obtain

$$
\begin{array}{rl}
2^{\ell \alpha} \mid f & * K *\left(D_{\left.2^{\ell} \phi\right)(x) \mid}\right. \\
& \lesssim \sum_{j \in \mathbb{Z}} 2^{-|j-\ell|(r-2-a-|\alpha|)} 2^{j \alpha} M_{a, 2^{-j}}^{*}(f, \widehat{\psi})(x) .
\end{array}
$$

Hence, it follows by [15, Lemma 2] that

$$
\begin{gathered}
\left\|\left(\sum_{\ell \in \mathbb{Z}}\left(2^{\ell \alpha}\left|f * K *\left(D_{2^{\ell}} \phi\right)(x)\right|\right)^{q}\right)^{1 / q}\right\|_{L^{p}} \\
\lesssim\left\|\left(\sum_{j \in \mathbb{Z}}\left(2^{j \alpha}\left|M_{a, 2^{-j}}^{*}(f, \widehat{\psi})\right|\right)^{q}\right)^{1 / q}\right\|_{L^{p}} .
\end{gathered}
$$


This together with (38) imply that $\|f * K\|_{\dot{F}_{p, q}^{\alpha}(G)} \lesssim\|f\|_{\dot{F}_{p, q}^{\alpha}(G)}$ for all $f \in \mathscr{Z}(G)$. Since $\mathscr{Z}(G)$ is dense in $\dot{F}_{p, q}^{\alpha}(G), f \mapsto f * K$ extends to an bounded operator on $\dot{F}_{p, q}^{\alpha}(G)$. This completes the proof of Theorem 18 .

Corollary 21. Let $\alpha \in \mathbb{R}, 0<p, q<\infty$, and let $k$ be a nonnegative integer. Then

$$
\|f\|_{\dot{F}_{p, q}^{\alpha}(G)} \sim \sum_{d(I)=k}\left\|X^{I} f\right\|_{\dot{F}_{p, q}^{\alpha-k}(G)} .
$$

Proof. Note that by the Poincaré-Birkhoff-Witt theorem (cf. [23, I.2.7]), the operators $X^{I}$ form a basis of the algebra of the left-invariant differential operators on $G$. By this fact and the stratification of $G$, it suffices to show that

$$
\|f\|_{\dot{F}_{p, q}^{\alpha}(G)} \sim \sum_{j=1}^{v}\left\|X_{j} f\right\|_{\dot{F}_{p, q}^{\alpha-1}(G)} .
$$

To this end, we first note that when restricted to Schwartz functions, $X_{j} \mathscr{L}^{-1 / 2}$ are convolution operators with distribution kernels homogeneous of degree $-\Delta$ and coincide with smooth functions in $G \backslash\{0\}$. This follows from the fact that the operator $\mathscr{L}^{-1 / 2}$ is a convolution operator whose distribution kernel is homogeneous of degree $-\Delta+1$ and coincides with a smooth function in $G \backslash\{0\}$ (see [2, Proposition 3.17]). Hence, by Theorem $18, X_{j} \mathscr{L}^{-1 / 2}$ extend to bounded operators on $\dot{F}_{p, q}^{\alpha}(G)$. From this fact and the lifting property (Theorem 7), we deduce that

$$
\begin{aligned}
\left\|X_{j} f\right\|_{\dot{F}_{p, q}^{\alpha-1}(G)} & =\left\|\left(X_{j} \mathscr{L}^{-1 / 2}\right) \mathscr{L}^{1 / 2} f\right\|_{\dot{F}_{p, q}^{\alpha-1}(G)} \\
& \lesssim\left\|\mathscr{L}^{1 / 2} f\right\|_{\dot{F}_{p, q}^{\alpha-1}(G)} \sim\|f\|_{\dot{F}_{p, q}^{\alpha}(G)} .
\end{aligned}
$$

Hence $\sum_{j=1}^{v}\left\|X_{j} f\right\|_{\dot{F}_{p, q}^{\alpha-1}(G)} \lesssim\|f\|_{\dot{F}_{p, q}^{\alpha}(G)}$. To see the converse, we need to use [2, Lemma 4.12], which asserts that there exists tempered distributions $K_{1}, \ldots, K_{v}$ homogeneous of degree $-\Delta+1$ and coinciding with smooth functions in $G \backslash\{0\}$ such that $f=\sum_{j=1}^{v}\left(X_{j} f\right) * K_{j}$ for all $f \in \mathcal{S}(G)$. By this result and Theorem 7, we have, at least for $f \in \mathscr{R}(G)\left(\subset \operatorname{Dom}\left(\mathscr{L}^{-1 / 2}\right)\right)$,

$$
\begin{aligned}
\|f\|_{\dot{F}_{p, q}^{\alpha}(G)} & =\left\|\mathscr{L}\left(\mathscr{L}^{-1 / 2} f\right)\right\|_{\dot{F}_{p, q}^{\alpha-1}(G)} \\
& =\left\|\mathscr{L}\left(f * R_{1}\right)\right\|_{\dot{F}_{p, q}^{\alpha-1}(G)} \\
& =\left\|\mathscr{L}\left(\sum_{j=1}^{v}\left(X_{j} f\right) * K_{j} * R_{1}\right)\right\|_{\dot{F}_{p, q}^{\alpha-1}(G)} \\
& =\left\|\sum_{j=1}^{v}\left(X_{j} f\right) * \mathscr{L}\left(K_{j} * R_{1}\right)\right\|_{\dot{F}_{p, q}^{\alpha-1}(G)},
\end{aligned}
$$

where $R_{1}$ is the convolution kernel of the operator $\mathscr{L}^{-1 / 2}$. As is indicated in [2, p. 190], $\mathscr{L}\left(K_{j} * R_{1}\right)$ are distributions homogeneous of degree $-\Delta$ and coincide with smooth functions away from 0 . Thus it follows by Theorem 18 that

$$
\left\|\left(X_{j} f\right) * \mathscr{L}\left(K_{j} * R_{1}\right)\right\|_{\dot{F}_{p, q}^{\alpha-1}(G)} \lesssim\left\|X_{j} f\right\|_{\dot{F}_{p, q}^{\alpha-1}(G)} .
$$

Inserting this into (93), we obtain $\|f\|_{\dot{F}_{p, q}^{\alpha}(G)} \lesssim \sum_{j=1}^{\nu}$ $\left\|X_{j} f\right\|_{\dot{F}_{p, q}^{\alpha-1}(G)}$ for all $f \in \mathscr{R}(G)$. Since $\mathscr{R}(G)$ is dense in $\dot{F}_{p, q}^{\alpha}(G)$, the latter inequality also holds for all $f \in \dot{F}_{p, q}^{\alpha}(G)$. This completes the proof.

\section{Appendix}

\section{Smooth Atomic Decomposition of $\dot{F}_{p, q}^{\alpha}(G)$}

In this appendix we show that homogeneous Triebel-Lizorkin spaces on stratified Lie groups admit smooth atomic decomposition. We follow the proof of [24, Theorem 6.6.3] with necessary modifications.

Equipped with Haar measure and the quasi-distance defined by the homogeneous norm, the group $G$ is a space of homogeneous type in the sense of Coifman and Weiss [25]. On such type of spaces, Christ [26] constructed a dyadic grid analogous to that of the Euclidean space as follows.

Lemma A.1. Let $G$ be a stratified Lie group. There exists a collection $\left\{Q_{\alpha}^{k}: k \in \mathbb{Z}, \alpha \in \mathscr{A}_{k}\right\}$ of open subsets of $G$, where $\mathscr{A}_{k}$ is some (possibly finite) index set, and constants $\delta \in(0,1)$ and $A_{1}, A_{2}>0$ such that

(i) $\left|G \backslash \bigcup_{\alpha \in \mathscr{A}_{k}} Q_{\alpha}^{k}\right|=0$ for each fixed $k$ and $Q_{\alpha}^{k} \cap Q_{\beta}^{k}=\emptyset$ if $\alpha \neq \beta$;

(ii) for any $\alpha, \beta, k, \ell$ with $\ell \geq k$, either $Q_{\beta}^{\ell} \subset Q_{\alpha}^{k}$ or $Q_{\beta}^{\ell} \cap$ $Q_{\alpha}^{k}=\emptyset$;

(iii) for each $(k, \alpha)$ and $\ell<k$, there exists a unique $\beta$ such that $Q_{\alpha}^{k} \subset Q_{\beta}^{\ell}$;

(iv) $\operatorname{diam}\left(Q_{\alpha}^{k}\right) \leq A_{1} \delta^{k}$, where $\operatorname{diam}\left(Q_{\alpha}^{k}\right):=\sup \left\{\left|x^{-1} y\right|\right.$ : $\left.x, y \in Q_{\alpha}^{k}\right\}$;

(v) each $Q_{\alpha}^{k}$ contains some ball $B\left(z_{\alpha}^{k}, A_{2} \delta^{k}\right)$, where $z_{\alpha}^{k} \in G$.

The set $Q_{\alpha}^{k}$ can be thought of as a dyadic cube with diameter roughly $\delta^{k}$ and centered at $z_{\alpha}^{k}$. We denote by $\mathscr{D}$ the family of all dyadic cubes on $G$. For $k \in \mathbb{Z}$, we set $\mathscr{D}_{k}=\left\{Q_{\alpha}^{k}\right.$ : $\left.\alpha \in \mathscr{A}_{k}\right\}$, so that $\mathscr{D}=\bigcup_{k \in \mathbb{Z}} \mathscr{D}_{k}$. For any dyadic cube $Q \in \mathscr{D}$, we denote by $z_{Q}$ the "center" of $Q$ and by $k_{Q}$ the unique integer $k$ such that $Q \in \mathscr{D}_{k}$.

Without loss of generality, in what follows we assume $\delta=$ $1 / 2$. Otherwise we need to replace $2^{j}$ in Definition 4 by $\delta^{-j}$, and also make some other necessary changes; see [27, pp. 9698] for more details.

Definition A.2. Let $Q$ be a dyadic cube and let $L$ be a nonnegative integer. A smooth function $a_{Q}$ on $G$ is called a smooth $L$-atom for $Q$ if it satisfies

(i) $a_{\mathrm{Q}}$ is supported in $B\left(z_{\mathrm{Q}},\left(\gamma\left(1+A_{1}\right) / A_{2}\right) 2^{-k_{\mathrm{Q}}}\right)$;

(ii) $\int a_{\mathrm{Q}}(x) P(x) d x=0$ for all $P \in \mathscr{P}_{L}$;

(iii) $\left|X^{I} a_{\mathrm{Q}}(x)\right| \leq|Q|^{-(d(I) / \Delta)-(1 / 2)}$ for all multi-indices $I$ with $|I| \leq L+1$. 
Definition A.3. Let $\alpha \in \mathbb{R}$ and $0<p, q<\infty$. The sequence space $\dot{f}_{p, q}^{\alpha}$ consists of all sequences $\left\{s_{\mathrm{Q}}\right\}_{\mathrm{Q} \in \mathscr{D}}$ such that the function

$$
g^{\alpha, q}\left(\left\{s_{\mathrm{Q}}\right\}_{\mathrm{Q}}\right)=\left(\sum_{\mathrm{Q} \in \mathscr{D}}\left(|\mathrm{Q}|^{-(\alpha / \Delta)-(1 / 2)}\left|s_{\mathrm{Q}}\right| \chi_{\mathrm{Q}}\right)^{q}\right)^{1 / q}
$$

is in $L^{p}(G)$. For such a sequence $s=\left\{s_{Q}\right\}_{Q}$ we set

$$
\|s\|_{\dot{f}_{p, q}^{\alpha}}=\left\|g^{\alpha, q}(s)\right\|_{L^{p}}
$$

The smooth atomic decomposition of homogeneous Triebel-Lizorkin spaces on stratified Lie groups can be stated as follows.

Theorem A.4. Let $\alpha \in \mathbb{R}, 0<p, q<\infty$, and let $L$ be $a$ nonnegative integer satisfying $L>[\Delta \max (1,1 / p, 1 / q)-\Delta-$ $\alpha]+1$. Then there is a constant $C_{\Delta, p, q, \alpha}$ such that for every sequence of smooth L-atoms $\left\{a_{\mathrm{Q}}\right\}_{\mathrm{Q} \in \mathscr{D}}$ and every sequence of complex scalars $\left\{s_{Q}\right\}_{Q \in \mathscr{D}}$ one has

$$
\left\|\sum_{\mathrm{Q} \in \mathscr{D}} s_{\mathrm{Q}} a_{\mathrm{Q}}\right\|_{\dot{F}_{p, q}^{\alpha}(G)} \leq C_{\Delta, p, q, \alpha}\left\|\left\{s_{\mathrm{Q}}\right\}_{\mathrm{Q} \in \mathscr{D}}\right\|_{\dot{f}_{p, q}^{\alpha}} .
$$

Conversely, there is a constant $C_{\Delta, p, q, \alpha}^{\prime}$ such that given any distribution $f \in \dot{F}_{p, q}^{\alpha}(G)$ and any $L \geq 0$, there exists a sequence of smooth L-atoms $\left\{a_{\mathrm{Q}}\right\}_{\mathrm{Q} \in \mathscr{D}}$ such that

$$
f=\sum_{\mathrm{Q} \in \mathscr{D}} s_{\mathrm{Q}} a_{\mathrm{Q}}
$$

where the sum converges in $\mathcal{S}^{\prime}(G) / \mathscr{P}$ and moreover

$$
\left\|\left\{s_{Q}\right\}_{Q}\right\|_{\dot{f}_{p, q}^{\alpha}} \leq C_{\Delta, p, q, \alpha}^{\prime}\|f\|_{\dot{F}_{p, q}^{\alpha}(G)} .
$$

Proof. Let $Q \in \mathscr{D}_{\ell}$ for some $\ell \in \mathbb{Z}$, and let $a_{\mathrm{Q}}$ be a smooth $L$-atom for $Q$. We set $a_{\mathrm{Q}}^{\prime}(x)=a_{\mathrm{Q}}\left(z_{\mathrm{Q}} x\right)$. Then $a_{\mathrm{Q}}^{\prime}$ is supported in $B\left(0,\left(\gamma\left(1+A_{1}\right) / A_{2}\right) 2^{-\ell}\right)$; moreover (since $1+2^{\ell}|y|: 1$ for $\left.y \in \operatorname{supp} a_{\mathrm{Q}}^{\prime}\right)$,

$$
\left|X^{I} a_{\mathrm{Q}}^{\prime}(y)\right| \leqslant 2^{-\ell \Delta / 2} \frac{2^{\ell d(I)+\ell \Delta}}{\left(1+2^{\ell}|y|\right)^{N}}, \quad \forall|I| \leq L+1,
$$

where $N$ can be chosen to be arbitrarily large. Set $a_{\mathrm{Q}}^{\prime \prime}(x)=$ $2^{-\ell \Delta} a_{\mathrm{Q}}^{\prime}\left(2^{-\ell} x\right)$, that is, $a_{\mathrm{Q}}^{\prime}=D_{2^{\ell}} a_{\mathrm{Q}}^{\prime \prime}$. Then the above inequality can be rewritten as

$$
\left|X^{I} a_{\mathrm{Q}}^{\prime \prime}(y)\right| \lesssim 2^{-\ell \Delta / 2} \frac{1}{(1+|y|)^{N}}, \quad \forall|I| \leq L+1 .
$$

Using this estimate, (73), and that $\operatorname{supp} a_{\mathrm{Q}}^{\prime \prime} \subset B(0,(\gamma(1+$ $\left.\left.A_{1}\right)\right) / A_{2}$ ), we also deduce that

$$
\begin{aligned}
\left|X^{I}\left(a_{\mathrm{Q}}^{\prime \prime}\right)^{\sim}(y)\right| & =\left|\left(Y^{I} a_{\mathrm{Q}}^{\prime \prime}\right)\left(y^{-1}\right)\right| \\
& \lesssim \sum_{\substack{|J| \leq|I| \\
d(J) \geq d(I)}}\left|y^{-1}\right|^{d(J)-d(I)}\left|\left(X^{J} a_{\mathrm{Q}}^{\prime \prime}\right)\left(y^{-1}\right)\right| \\
& \leqslant 2^{-\ell \Delta / 2} \frac{1}{(1+|y|)^{N}}, \quad \forall|I| \leq L+1 .
\end{aligned}
$$

Thus, it follows from Lemma 2 that

$$
\begin{aligned}
\left|a_{\mathrm{Q}}^{\prime} *\left(D_{2^{j}} \phi\right)(x)\right| & =\left|\left(D_{2^{\ell}} a_{\mathrm{Q}}^{\prime \prime}\right) *\left(D_{2^{j}} \phi\right)(x)\right| \\
& \leq 2^{-\ell \Delta / 2} 2^{-|j-\ell| L} \frac{2^{-(j \wedge \ell) \Delta}}{1+\left(2^{j \wedge \ell}\left|z_{\mathrm{Q}}^{-1} x\right|\right)^{N}}
\end{aligned}
$$

where $\widehat{\phi} \in \mathscr{A}$, and $N$ can be taken to be arbitrarily large. Consequently,

$$
\begin{aligned}
\mid a_{\mathrm{Q}} * & \left(D_{2^{j}} \phi\right)(x) \mid \\
& =\left|a_{\mathrm{Q}}^{\prime} *\left(D_{2^{j}} \phi\right)\left(z_{\mathrm{Q}}^{-1} x\right)\right| \\
& \leqslant 2^{-\ell \Delta / 2} 2^{-|j-\ell| L} \frac{2^{-(j \wedge \ell) \Delta}}{1+\left(2^{j \wedge \ell}\left|z_{\mathrm{Q}}^{-1} x\right|\right)^{N}}
\end{aligned}
$$

Note that if $r \in(0,1]$ and $N>\Delta / r$ then we have

$\sum_{Q \in \mathscr{D}_{\ell}} \frac{\left|s_{Q}\right|}{\left(1+2^{j \wedge \ell}\left|z_{Q}^{-1} x\right|\right)^{N}}$

$$
\begin{aligned}
& \leq\left(\sum_{Q \in \mathscr{D}_{\ell}} \frac{\left|s_{\mathrm{Q}}\right|^{r}}{\left(1+2^{j \wedge \ell}\left|z_{\mathrm{Q}}^{-1} x\right|\right)^{N r}}\right)^{1 / r} \\
& =\left(\int_{G} \sum_{Q \in \mathscr{D}_{\ell}} \frac{\left|s_{\mathrm{Q}}\right|^{r}|Q|^{-1} \chi_{\mathrm{Q}}(z)}{\left(1+2^{j \wedge \ell}\left|z_{\mathrm{Q}}^{-1} x\right|\right)^{N r}} d z\right)^{1 / r} \\
& \lesssim 2^{\ell \Delta / r}\left\{\int_{\left|x^{-1} z\right| \leq\left(2 \gamma A_{1}\right) 2^{-(j \wedge \ell)}}\left(\sum_{\mathrm{Q} \in \mathscr{D}_{\ell}} \frac{\left|s_{\mathrm{Q}}\right|^{r} \chi_{\mathrm{Q}}(z)}{\left(1+2^{j \wedge \ell}\left|z_{\mathrm{Q}}^{-1} x\right|\right)^{N r}}\right) d z\right. \\
& +\sum_{k=0}^{\infty} \int_{\left(2 \gamma A_{1}\right) 2^{k} 2^{-(j \wedge \ell)} \leq\left|x^{-1} z\right| \leq\left(2 \gamma A_{1}\right) 2^{k+1} 2^{-(j \wedge \ell)}} \\
& \left.\times\left(\sum_{Q \in \mathscr{D}_{\ell}} \frac{\left|s_{\mathrm{Q}}\right|^{r} \chi_{\mathrm{Q}}(z)}{\left(1+2^{j \wedge \ell}\left|z_{\mathrm{Q}}^{-1} x\right|\right)^{N r}}\right) d z\right\}^{1 / r} \\
& \lesssim 2^{\ell \Delta / r}\left\{\int_{\left|x^{-1} z\right| \leq\left(2 \gamma A_{1}\right) 2^{-(j \wedge \ell)}}\left(\sum_{Q \in D_{\ell}}\left|s_{Q}\right|^{r} \chi_{Q}(z)\right) d z\right. \\
& +\sum_{k=0}^{\infty}\left(1+A_{1} 2^{k}\right)^{-N r}
\end{aligned}
$$




$$
\begin{aligned}
& \times \int_{\left(2 \gamma A_{1}\right) 2^{k} 2^{-(j \wedge \ell)} \leq\left|x^{-1} z\right| \leq\left(2 \gamma A_{1}\right) 2^{k+1} 2^{-(j \wedge \ell)}} \\
& \left.\times\left(\sum_{Q \in \mathscr{D}_{\ell}}\left|s_{\mathrm{Q}}\right|^{r} \chi_{\mathrm{Q}}(z)\right) d z\right\}^{1 / r} \\
& \lesssim 2^{\ell \Delta / r}\left\{\frac{2^{-(j \wedge \ell) \Delta}}{\left|B\left(x, 2 \gamma A_{1} 2^{-(j \wedge \ell)}\right)\right|} \int_{B\left(x, 2 \gamma A_{1} 2^{-(j \wedge \ell)}\right)}\right. \\
& \times\left(\sum_{Q \in \mathscr{D}_{\ell}}\left|s_{\mathrm{Q}}\right|^{r} \chi_{\mathrm{Q}}(z)\right) d z \\
& +\sum_{k=0}^{\infty}\left(1+A_{1} 2^{k}\right)^{-N r} \frac{2^{(k+1) \Delta} 2^{-(j \wedge \ell) \Delta}}{\left|B\left(x, 2 \gamma A_{1} 2^{k+1} 2^{-(j \wedge \ell)}\right)\right|} \\
& \times \int_{B\left(x, 2 \gamma A_{1} 2^{k+1} 2^{-(j \wedge \ell)}\right)} \\
& \left.\times\left(\sum_{Q \in \mathscr{D}_{\ell}}\left|s_{\mathrm{Q}}\right|^{r} \chi_{\mathrm{Q}}(z)\right) d z\right\}^{1 / r} \\
& \leq 2^{\ell \Delta / r}\left\{2^{-(j \wedge \ell) \Delta}+\sum_{k=0}^{\infty}\left(1+A_{1} 2^{k}\right)^{-N r} 2^{(k+1) \Delta} 2^{-(j \wedge \ell) \Delta}\right\}^{1 / r} \\
& \times\left\{M\left(\sum_{Q \in D_{\ell}}\left|s_{Q}\right|^{r} \chi_{Q}\right)(x)\right\}^{1 / r} \\
& \lesssim 2^{\ell \Delta / r} 2^{-(j \wedge \ell) \Delta / r}\left\{M\left(\sum_{\mathrm{Q} \in \mathscr{D}_{\ell}}\left|s_{\mathrm{Q}}\right|^{r} \chi_{\mathrm{Q}}\right)(x)\right\}^{1 / r} \\
& =2^{\max (\ell-j, 0) \Delta / r}\left\{M\left(\sum_{Q \in \mathscr{D}_{\ell}}\left|s_{\mathrm{Q}}\right|^{r} \chi_{\mathrm{Q}}\right)(x)\right\}^{1 / r} .
\end{aligned}
$$

Here we used the fact that for $z \in Q$ and $\left|x^{-1} z\right| \geq$ $\left(2 \gamma A_{1}\right) 2^{k} 2^{-(j \wedge \ell)}$ one has $\left(1+2^{j \wedge \ell}\left|z_{\mathrm{Q}}^{-1} x\right|\right)^{N r} \geq\left(1+A_{1} 2^{-k}\right)^{N r}$, which can be easily verified by using Lemma 19 (iv) and the quasi-triangle inequality satisfied by the homogeneous norm. The above estimate and (A.10), along with the argument in [24, pp. 80-81], yield (A.3).

Now we show the converse statement of the theorem. By Lemma 20 , there exists $\widehat{\zeta} \in \mathcal{S}\left(\mathbb{R}^{+}\right)$such that $|\widehat{\zeta}(\lambda)| \geq$ $C>0$ for $\lambda \in\left[2^{-2\left(k_{0}+1\right)}, 2^{-2\left(k_{0}-1\right)}\right]$ for some (large) positive integer $k_{0}$, and that $\zeta$ is supported in $B(0,1)$ and has vanishing moments of order $L$. Then it is possible to find a function $\widehat{\psi} \in S\left(\mathbb{R}^{+}\right)$with the properties that supp $\psi \subset$ $\left[2^{-2\left(k_{0}+1\right)}, 2^{-2\left(k_{0}-1\right)}\right],|\widehat{\psi}(\lambda)|>0$ for $\lambda \in\left(2^{-2\left(k_{0}+1\right)}, 2^{-2\left(k_{0}-1\right)}\right)$, and $\sum_{j \in \mathbb{Z}} \widehat{\psi}\left(2^{-2 j} \lambda\right) \widehat{\zeta}\left(2^{-2 j} \lambda\right)=1$ for $\lambda \in \mathbb{R}^{+}$. Note that $\widehat{\psi}\left(2^{-2 k_{0}} \cdot\right) \in \mathscr{A}$. Given $f \in \dot{F}_{p, q}^{\alpha}(G)$, it follows from Lemma 3 that

$$
f=\sum_{j \in \mathbb{Z}} f *\left(D_{2^{j}} \psi\right) *\left(D_{2^{j}} \zeta\right)
$$

with the convergence in $\mathcal{S}^{\prime}(G) / \mathscr{P}$. Let us decompose $f$ as

$$
\begin{aligned}
f & =\sum_{j \in \mathbb{Z}} \sum_{Q \in \mathscr{D}_{j}} \int_{\mathrm{Q}} f *\left(D_{2^{j}} \psi\right)(y)\left(D_{2^{j}} \zeta\right)\left(y^{-1} x\right) d y \\
& =\sum_{j \in \mathbb{Z}} \sum_{Q_{Q} \in \mathscr{D}_{j}} s_{\mathrm{Q}} a_{\mathrm{Q}},
\end{aligned}
$$

where we have set, for $Q \in \mathscr{D}_{j}$,

$$
\begin{gathered}
s_{\mathrm{Q}} \equiv|Q|^{1 / 2} \sup _{y \in \mathrm{Q}}\left|f *\left(D_{2^{j}} \psi\right)(y)\right| \sup _{|I| \leq L+1}\left\|X^{I} \zeta\right\|_{L^{1}}, \\
a_{\mathrm{Q}}(x) \equiv \frac{1}{s_{\mathrm{Q}}} \int_{\mathrm{Q}} f *\left(D_{2^{j}} \psi\right)(y)\left(D_{2^{j}} \zeta\right)\left(y^{-1} x\right) d y .
\end{gathered}
$$

It is straightforward to verify that $a_{\mathrm{Q}}$ is supported in $B\left(z_{\mathrm{Q}},\left(\gamma\left(1+A_{1}\right) / A_{2}\right) 2^{-j}\right)$, and that $a_{\mathrm{Q}}$ has vanishing moments of the same order as $\zeta$. Moreover, for $Q \in \mathscr{D}_{j}$ we have

$$
\begin{aligned}
\left|X^{I} a_{\mathrm{Q}}\right| & \leq \frac{2^{\Delta j} 2^{d(I) j}}{s_{Q}} \int_{Q}\left|f *\left(D_{2^{j}} \psi\right)(y)\left(X^{I} \zeta\right)\left(2^{j}\left(y^{-1} x\right)\right)\right| d y \\
& \leq \frac{2^{d(I) j}}{s_{Q}}\left\|\left(X^{I} \zeta\right)\right\|_{L^{1}} \sup _{y \in Q}\left|f *\left(D_{2^{j}} \psi\right)(y)\right| \\
& \leq|Q|^{-(d(I) / \Delta)-(1 / 2)}
\end{aligned}
$$

for all $|I| \leq L+1$. Hence the function $a_{\mathrm{Q}}$ a smooth $L$-atom for Q. Choose any $\lambda>\Delta / \min \{p, q\}$. We note that

$$
\begin{aligned}
\sum_{Q \in \mathscr{D}_{j}}\left(|Q|^{-(\alpha / \Delta)-(1 / 2)} s_{\mathrm{Q}} \chi_{\mathrm{Q}}(x)\right)^{q} \\
\quad \sim \sum_{\mathrm{Q}_{\in \mathscr{D}_{j}}}\left(2^{j \alpha} \sup _{y \in \mathrm{Q}}\left|f *\left(D_{2^{j}} \psi\right)(y)\right| \chi_{\mathrm{Q}}(x)\right)^{q} \\
\quad \lesssim \sup _{y \in B\left(x, A_{1} 2^{-j}\right)}\left[2^{j \alpha}\left|f *\left(D_{2^{j}} \psi\right)(y)\right|\right]^{q} \\
\quad \lesssim\left[2^{j \alpha} M_{\lambda, 2^{-j}}^{*}(f, \widehat{\psi})(x)\right]^{q} .
\end{aligned}
$$

We thus obtain, using (38),

$$
\left\|\left\{s_{Q}\right\}_{Q}\right\|_{\dot{f}_{p, q}^{\alpha}} \lesssim\left\|\left(\sum_{j \in \mathbb{Z}}\left|2^{j \alpha} M_{\lambda, 2^{-j}}^{*}(f, \widehat{\psi})\right|^{q}\right)^{1 / q}\right\|_{L^{p}} \lesssim\|f\|_{\dot{F}_{p, q}^{\alpha}(G)} .
$$

This proves (A.5). 


\section{Acknowledgments}

The author would like to thank Professor Hitoshi Arai for his patient guidance and constant support. He is also grateful to Professor Yoshihiro Sawano for his valuable comments.

\section{References}

[1] Y. Han, D. Müller, and D. Yang, "A theory of Besov and TriebelLizorkin spaces on metric measure spaces modeled on CarnotCarathéodory spaces," Abstract and Applied Analysis, vol. 2008, Article ID 893409, 250 pages, 2008.

[2] G. B. Folland, "Subelliptic estimates and function spaces on nilpotent Lie groups," Arkiv för Matematik, vol. 13, no. 2, pp. 161207, 1975.

[3] G. B. Folland and E. M. Stein, Hardy Spaces on Homogeneous Groups, vol. 28 of Mathematical Notes, Princeton University Press, Princeton, NJ, USA, 1982.

[4] K. Saka, "Besov spaces and Sobolev spaces on a nilpotent Lie group," The Tôhoku Mathematical Journal, vol. 31, no. 4, pp. 383437, 1979.

[5] H. Führ and A. Mayeli, "Homogeneous Besov spaces on stratified Lie groups and their wavelet characterization," Journal of Function Spaces and Applications, vol. 2012, Article ID 523586, 41 pages, 2012.

[6] A. W. Knapp and E. M. Stein, "Intertwining operators for semisimple groups," Annals of Mathematics, vol. 93, pp. 489$578,1971$.

[7] A. Korányi and S. Vági, "Singular integrals on homogeneous spaces and some problems of classical analysis," Annali della Scuola Normale Superiore di Pisa, vol. 25, pp. 575-648, 1971.

[8] M. Christ, " $L^{p}$ bounds for spectral multipliers on nilpotent groups," Transactions of the American Mathematical Society, vol. 328, no. 1, pp. 73-81, 1991.

[9] A. Hulanicki, "A functional calculus for Rockland operators on nilpotent Lie groups," Studia Mathematica, vol. 78, no. 3, pp. 253-266, 1984.

[10] D. Geller and A. Mayeli, "Continuous wavelets and frames on stratified Lie groups. I," The Journal of Fourier Analysis and Applications, vol. 12, no. 5, pp. 543-579, 2006.

[11] M. Frazier and B. Jawerth, "A discrete transform and decompositions of distribution spaces," Journal of Functional Analysis, vol. 93, no. 1, pp. 34-170, 1990.

[12] A. Bonfiglioli, E. Lanconelli, and F. Uguzzoni, Stratified Lie Groups and Potential Theory for Their Sub-Laplacians, Springer Monographs in Mathematics, Springer, Berlin, Germany, 2007.

[13] M. Reed and B. Simon, Methods of Modern Mathematical Physics. I: Functional Analysis, Academic Press, New York, NY, USA, 2nd edition, 1980.

[14] L. Grafakos, L. Liu, and D. Yang, "Vector-valued singular integrals and maximal functions on spaces of homogeneous type," Mathematica Scandinavica, vol. 104, no. 2, pp. 296-310, 2009.

[15] V. S. Rychkov, "On a theorem of Bui, Paluszyński, and Taibleson," Proceedings of the Steklov Institute of Mathematics, vol. 227, pp. 280-292, 1999.

[16] H. Triebel, Theory of Function Spaces, vol. 78 of Monographs in Mathematics, Birkhäuser, Basel, Switzerland, 1983.

[17] W. Rudin, Functional Analysis, International Series in Pure and Applied Mathematics, McGraw-Hill, New York, NY, USA, 2nd edition, 1991.
[18] L. Päivärinta, "Equivalent quasinorms and Fourier multipliers in the Triebel spaces $F_{p, q}^{s}$ " Mathematische Nachrichten, vol. 106, pp. 101-108, 1982.

[19] J.-O. Strömberg and A. Torchinsky, Weighted Hardy Spaces, vol. 1381 of Lecture Notes in Mathematics, Springer, Berlin, Germany, 1989.

[20] M. Bownik, B. Li, D. Yang, and Y. Zhou, "Weighted anisotropic product Hardy spaces and boundedness of sublinear operators," Mathematische Nachrichten, vol. 283, no. 3, pp. 392-442, 2010.

[21] E. M. Stein, Harmonic Analysis: Real-Variable Methods, Orthogonality, and Oscillatory Integrals, vol. 43 of Princeton Mathematical Series, Princeton University Press, Princeton, NJ, USA, 1993.

[22] L. Grafakos and X. Li, "Bilinear operators on homogeneous groups," Journal of Operator Theory, vol. 44, no. 1, pp. 63-90, 2000.

[23] N. Bourbaki, Groupes et Algèbres de Lie, (Eléments de Math., Fasc. 26 et 37), Chapter I-III, Hermann, Paris, France, 1960 and 1972.

[24] L. Grafakos, Modern Fourier Analysis, vol. 250 of Graduate Texts in Mathematics, Springer, New York, NY, USA, 2nd edition, 2009.

[25] R. R. Coifman and G. Weiss, "Extensions of Hardy spaces and their use in analysis," Bulletin of the American Mathematical Society, vol. 83, no. 4, pp. 569-645, 1977.

[26] M. Christ, "A $T(b)$ theorem with remarks on analytic capacity and the Cauchy integral," Colloquium Mathematicum, vol. 6061, no. 2, pp. 601-628, 1990.

[27] Y. S. Han and E. T. Sawyer, "Littlewood-Paley theory on spaces of homogeneous type and the classical function spaces," Memoirs of the American Mathematical Society, vol. 110, no. 530, pp. 1-126, 1994. 


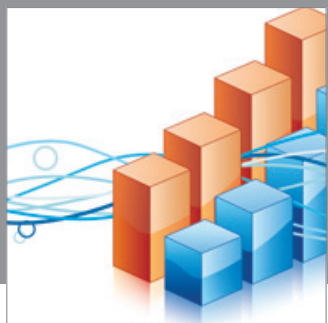

Advances in

Operations Research

mansans

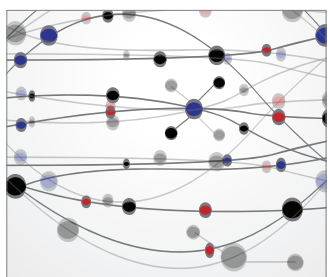

The Scientific World Journal
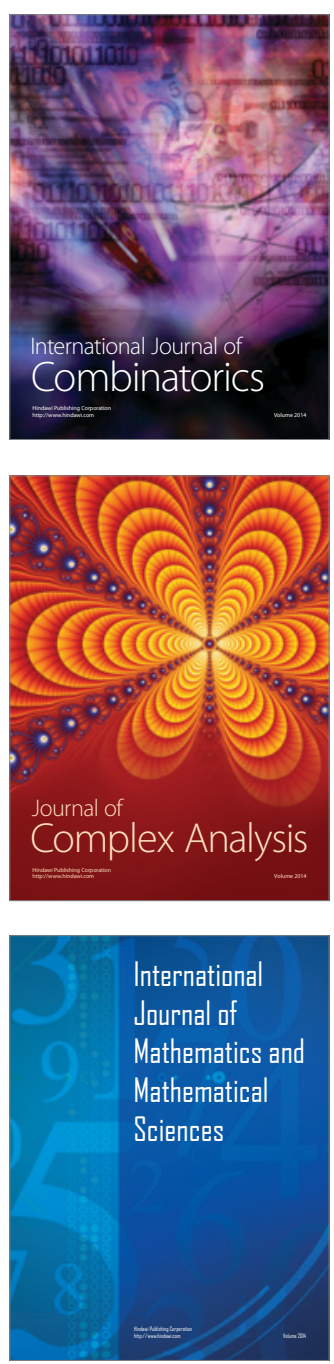
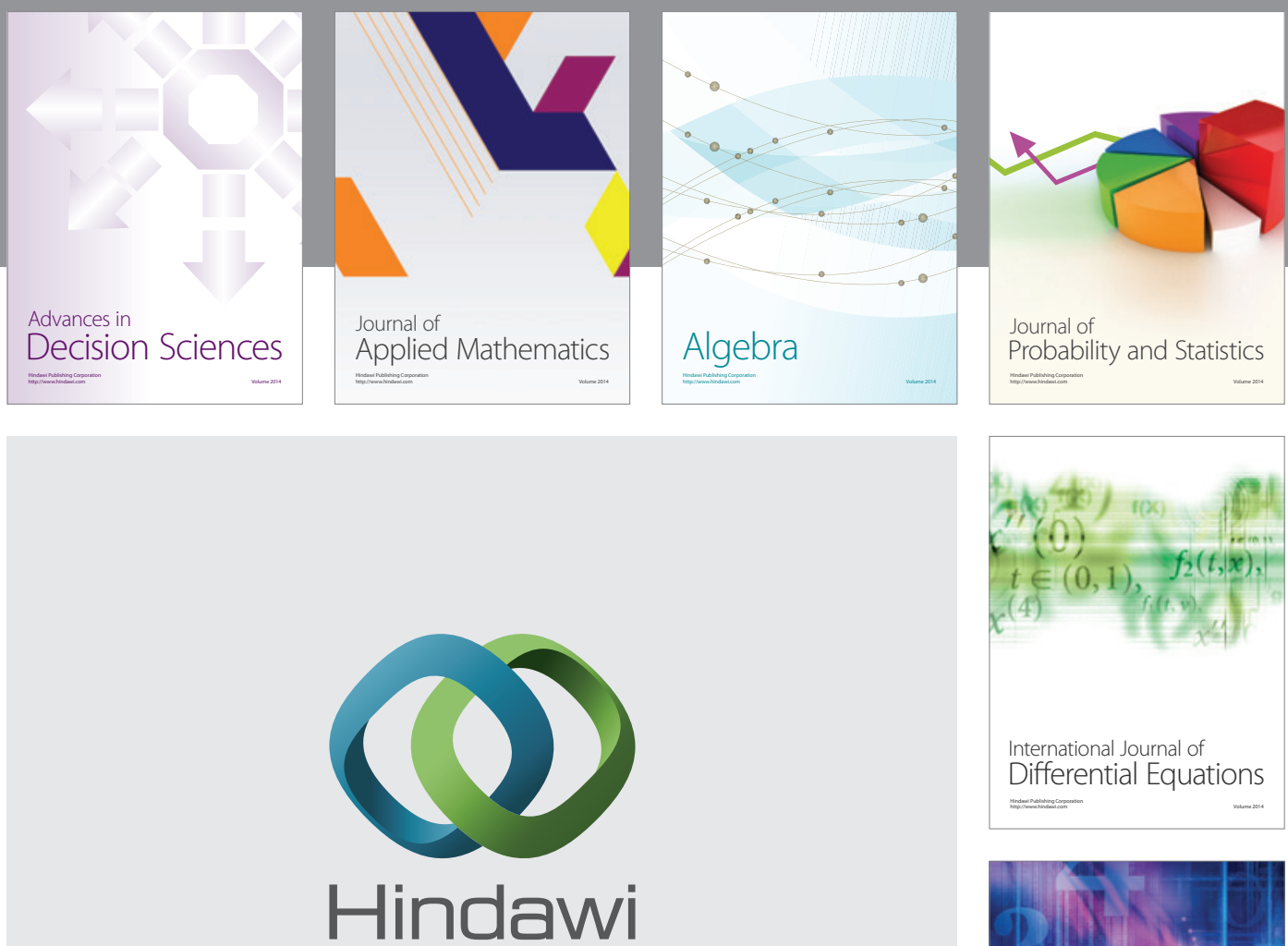

Submit your manuscripts at http://www.hindawi.com
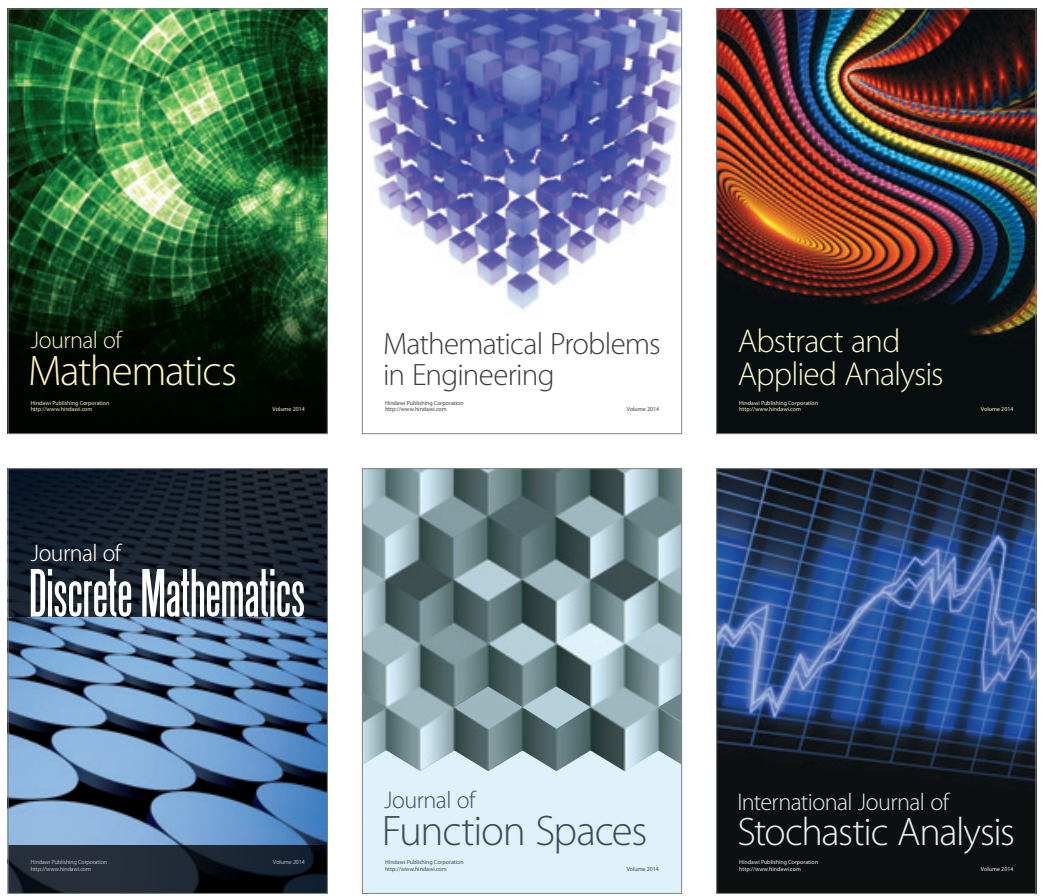

Journal of

Function Spaces

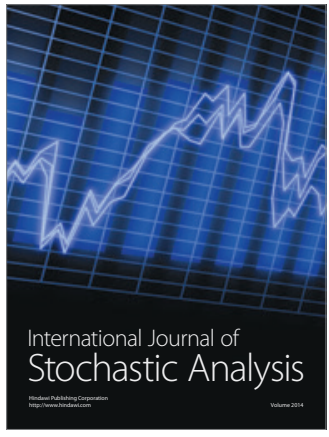

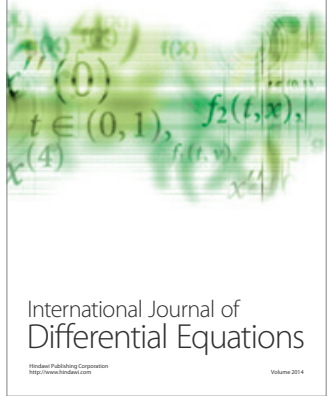
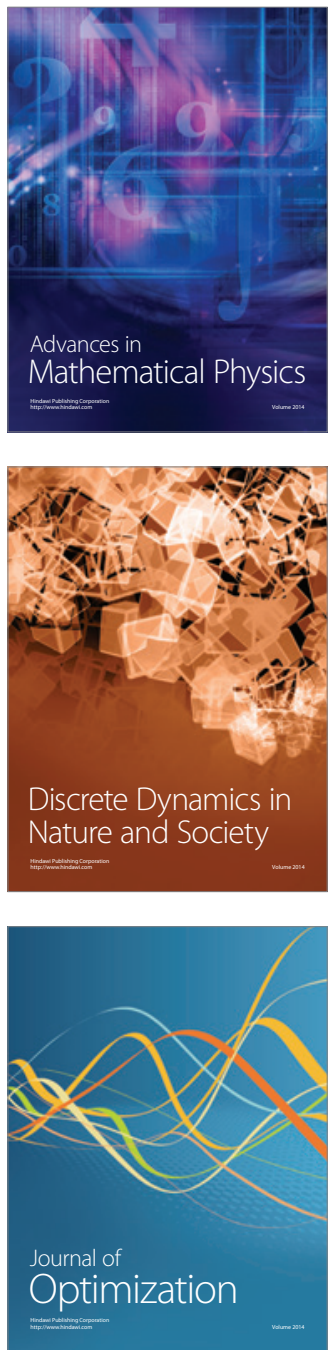